\title{
C-VALUED FREE PROBABILITY ON A GRAPH VON NEUMANN ALGEBRA
}

\author{
Ilwoo CHO
}

\begin{abstract}
In [6] and [7], we introduced graph von Neumann algebras which are the (groupoid) crossed product algebras of von Neumann algebras and graph groupoids via groupoid actions. We showed that such crossed product algebras have the graph-depending amalgamated reduced free probabilistic properties. In this paper, we will consider a scalarvalued $W^{*}$-probability on a given graph von Neumann algebra. We show that a diagonal graph $W^{*}$-probability space (as a scalar-valued $W^{*}$ probability space) and a graph $W^{*}$-probability space (as an amalgamated $W^{*}$-probability space) are compatible. By this compatibility, we can find the relation between amalgamated free distributions and scalar-valued free distributions on a graph von Neumann algebra. Under this compatibility, we observe the scalar-valued freeness on a graph von Neumann algebra.
\end{abstract}

In [6], we introduced a graph von Neumann algebra $\mathbb{M}_{G}=M \times_{\alpha} \mathbb{G}$ over a fixed von Neumann algebra $M$. This von Neumann algebra $\mathbb{M}_{G}$ is a crossed product algebra of $M$ and a graph groupoid $\mathbb{G}$ induced by a given countable directed graph $G$ via a groupoid $\alpha$ of $\mathbb{G}$, acting on $M$. We observed the amalgamated reduced free probabilistic properties of $\mathbb{M}_{G}$, where the reduction is depending on the given graph $G$. Such amalgamated free probabilistic data of a graph von Neumann algebra $\mathbb{M}_{G}$ makes us deal with $\mathbb{M}_{G}$ as a reduced free product of certain amalgamated free blocks. Since the free blocks are characterized in [7], we are in good situation to study such crossed product algebras, i.e., we can concentrate on the combinatorial data (the admissibility) of the given graph $G$ and the amalgamated free blocks to study $\mathbb{M}_{G}$.

Let $M$ be a von Neumann algebra and $\operatorname{tr}: M \rightarrow \mathbb{C}$ be a trace on $M$, as a continuous linear functional on $M$ satisfying that (i) $\operatorname{tr}\left(m^{*}\right)=\overline{\operatorname{tr}(m)}$ for all $m \in$ $M$, where $\bar{z}$ is the conjugate of $z$ for all $z \in \mathbb{C}$, and (ii) $\operatorname{tr}\left(m_{1} m_{2}\right)=\operatorname{tr}\left(m_{2} m_{1}\right)$ for all $m_{1}, m_{2} \in M$. The pair $(M, \operatorname{tr})$ is called a tracial (scalar-valued or $\mathbb{C}$-valued) $W^{*}$-probability space.

Received September 10, 2008; Revised October 7, 2008.

2000 Mathematics Subject Classification. 05C62, 05C90, 17A50, 18B40, 46K10, 47A67, 47A99, 47B99.

Key words and phrases. graph groupoids, crossed products, graph von Neumann algebras, $w^{*}$-probability spaces. 
In this paper, we will study graph von Neumann algebras in scalar-valued $W^{*}$-probability point of view. Recall that, in $[5,6,7,8]$, we have studied graph von Neumann algebras in amalgamated $W^{*}$-probability point of view. We consider a graph von Neumann algebra $\mathbb{M}_{G}=M \times_{\alpha} \mathbb{G}$, where $(M, \operatorname{tr})$ is a tracial $W^{*}$-probability space with its fixed trace $\operatorname{tr}$, with a certain trace $\tau: \mathbb{M}_{G} \rightarrow \mathbb{C}$, induced by the trace tr on $M$, and we observe the scalar-valued $W^{*}$-probability space $\left(\mathbb{M}_{G}, \tau\right)$. By studying such structure, we can get a new information about a graph von Neumann algebra $\mathbb{M}_{G}$. The main purpose of this paper is to find the differences or the similarities between the amalgamated free properties of $\mathbb{M}_{G}$ (over its $M$-diagonal subalgebra $\mathbb{D}_{G}$ ) and the scalar-valued free properties of $\mathbb{M}_{G}$ (over $\mathbb{C}$ ).

\section{Introduction}

The study of finite or infinite directed graphs in free probability theory entails two abstractions: the notion of groupoids and certain class of von Neumann algebras, the weakly closed algebras of bounded operators in Hilbert space. While the classical and analogous constructions, going back to von Neumann, involve crossed products of von Neumann algebras by groups. The study of directed graphs entail instead another crossed product construction, now with groupoids rather than groups. Further the study of new invariants for graphs and graph von Neumann algebras replies on certain tracial states. A main purpose of this paper is to find them and to prove the results needed in the analysis of graph von Neumann algebras. Our main theorems are in Sections 3 and 4 . Once the tools have been built, we are able in Theorem 4.1 to compare two levels of the theory; operator-valued and scalar-valued. The later is taking the form of a certain diagonal algebra in a groupoid crossed product von Neumann algebra.

Free probability theory was initiated by D. Voiculescu in the 1980 's. While the initial motivation was an old problem of von Neumann, now known as the free group factors $*$-isomorphism problem. The noncommutative theory has since been adopted by probabilists, especially, in the study of random matrices from quantum physics, and more specially the asymptotic distribution of the matrix entries (as random variables) in large matrices. The original von Neumann problem however is still open (even through there are partial results), viz; given a free group $F_{n}$ with $n$-generators, we can consider the von Neumann algebra generated by the group algebra of $F_{n}$ for $n \in \mathbb{N} \backslash\{1\}$. The $*$-isomorphism problem asks if the von Neumann factors are $*$-isomorphic for different numbers of generators. Radulsecu showed the alternative $*$-isomorphism theorem: either (i) or (ii) holds, where

(i) $L\left(F_{n}\right) \stackrel{* \text {-isomorphic }}{=} L\left(F_{m}\right)$, where $n, m \in(\mathbb{N} \backslash\{1\}) \cup\{\infty\}$,

(ii) $L\left(F_{n}\right) \stackrel{* \text {-isomorphic }}{\neq} L\left(F_{m}\right)$, whenever $n \neq m$ in $\mathbb{N} \backslash\{1\}$. 
Spinoff problems are of current and independent interest, for example, because of connections to random matrix theory, to analysis of direct graphs, and to combinatorics, represents entations of symmetric groups, and large deviations.

Random variables are measurable functions on a probability space, so they form abelian algebras under multiplication. By contrast, algebras in quantum theory are highly non-abelian, and a problem of Wigner suggested a new notion of independence for noncommutative probability. Voiculescu made it precise as follows: In the noncommutative theory, the random variables lie instead in a unital $C^{*}$-algebra or a von Neumann algebra. The algebra comes equipped with a noncommutative expectation.

As in [6], we define the $M$-diagonal subalgebra $\mathbb{D}_{G}$ of $\mathbb{M}_{G}$ by

$$
\underset{v \in V(G)}{\oplus}\left(M \cdot L_{v}\right) .
$$

Notice that each direct summand is $*$-isomorphic to the von Neumann algebra $M$ for all $v \in V(G)$, where $V(G)$ is the vertex set of $G$ (See below). Therefore, we can naturally define a trace

$$
\operatorname{tr}_{v}: M \cdot L_{v} \longrightarrow \mathbb{C} \cdot L_{v}
$$

by

$$
\operatorname{tr}_{v}\left(m L_{v}\right) \stackrel{\text { def }}{=} \operatorname{tr}(m) L_{v} \text { for all } m L_{v} \in M \cdot L_{v}
$$

for all $v \in V(G)$, where $\operatorname{tr}$ is the given trace on $M$. Let's construct a continuous linear functional

$$
\operatorname{Tr}: \mathbb{D}_{G} \longrightarrow \mathbb{C}
$$

by

$$
\operatorname{Tr}\left(\sum_{v \in V(G)} m_{v} L_{v}\right) \stackrel{\text { def }}{=} \sum_{v \in V(G)} \operatorname{tr}_{v}\left(m_{v} L_{v}\right)
$$

for all $\sum_{v \in V(G)} m_{v} L_{v} \in \mathbb{D}_{G}$. Then this linear functional $\operatorname{Tr}$ on $\mathbb{D}_{G}$ satisfies $\operatorname{Tr}\left(d^{*}\right)=\overline{\operatorname{Tr}(d)}$ for all $d \in \mathbb{D}_{G}$ and $\operatorname{Tr}\left(d_{1} d_{2}\right)=\operatorname{Tr}\left(d_{2} d_{1}\right)$ for all $d_{1}, d_{2} \in \mathbb{D}_{G}$, and hence it is a trace on the $M$-diagonal subalgebra $\mathbb{D}_{G}$ of $\mathbb{M}_{G}$. Now, we can define a continuous linear functional on $\mathbb{M}_{G}$

$$
\tau: \mathbb{M}_{G} \longrightarrow \mathbb{C}
$$

by

$$
\tau \stackrel{\text { def }}{=} \operatorname{Tr} \circ E \text {. }
$$

Clearly, the pair $\left(\mathbb{M}_{G}, \tau\right)$ is a $W^{*}$-probability space. This $W^{*}$-probability space is called the diagonal graph $W^{*}$-probability space. Notice that, in general, the linear functional $\tau$ is not a trace on $\mathbb{M}_{G}$ even though $\operatorname{Tr}$ is a well-defined trace on $\mathbb{D}_{G}$, because of a groupoid action $\alpha$. That's why we sometimes call the pair $\left(\mathbb{M}_{G}, \tau\right)$, the diagonal non-tracial graph $W^{*}$-probability space (See Section 2). 
However, we can consider the very special cases. Define a $W^{*}$-subalgebra $M^{\alpha}$ of $M$ by

$$
M^{\alpha} \stackrel{\text { def }}{=} v N\left(\left\{m \in M: \alpha_{w}(m)=m, \forall w \in \mathbb{G}\right\}\right) .
$$

If $M^{\alpha}=M$, the von Neumann algebra $M$ is said to be $\alpha$-fixed. Whenever $M$ is $\alpha$-fixed, the linear functional $\tau=\operatorname{tr} \circ E$ on $\mathbb{M}_{G}$ is a trace. For example, if $\left.\alpha_{w}\right|_{M}=1_{M}$ for all $w \in \mathbb{G}$, then $M$ is $\alpha$-fixed (only for $\alpha$ ). Remark that the $\alpha$-fixed property of $M$ is totally depending on the given groupoid action $\alpha$, unless $M$ is $*$-isomorphic to $\mathbb{C}$. In such $\alpha$-fixed case, the pair $\left(\mathbb{M}_{G}, \tau\right)$ is a tracial $W^{*}$-probability space.

The main purpose of this paper is to consider the (scalar-valued or $\mathbb{C}$-valued) free probabilistic property of our graph von Neumann algebra $\mathbb{M}_{G}$ with respect to the linear functional $\tau=\operatorname{Tr} \circ E$. Also, we will investigate the relations between these scalar-valued free probabilistic information of $\mathbb{M}_{G}$ and the amalgamated (or $\mathbb{D}_{G}$-valued) reduced free probabilistic information of $\mathbb{M}_{G}$ (observed in $[6,7])$. We can easily verify that the free distributional data of arbitrary

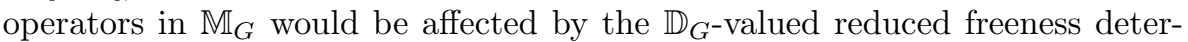
mined by the canonical conditional expectation $E$ from $\mathbb{M}_{G}$ onto $\mathbb{D}_{G}$ and by the scalar-valued free distributional data depending on $\operatorname{Tr}$ and $\operatorname{tr}$ on $\mathbb{D}_{G}$ and $M$, respectively, by the very definition of $\tau$ on $\mathbb{M}_{G}$. This observation also leads us to verify that the free probabilistic information on $\mathbb{M}_{G}$ with respect to $\tau$ would have a similar reduction depending on the reduction on the graph groupoid $\mathbb{G}$ of $G$, by the conditional expectation $E$. Also, we can verify that if $\tau$ is a trace, we will have much familiar structure than the non-tracial cases (See [3]).

By the very definition, the $M$-diagonal graph $W^{*}$-probability space $\left(\mathbb{M}_{G}\right.$, $E$ ), in the sense of [6] and [7], and the diagonal non-tracial (or tracial) graph $W^{*}$-probability space $\left(\mathbb{M}_{G}, \tau\right)$ are compatible in the sense that

$$
\tau\left(\sum_{w \in \mathbb{G}} m_{w} L_{w}\right)=\tau\left(E\left(\sum_{w \in \mathbb{G}} m_{w} L_{w}\right)\right)
$$

for all $\sum_{w \in \mathbb{G}} m_{w} L_{w} \in \mathbb{M}_{G}$.

We will consider such free distributional properties of graph von Neumann algebras determined by the compatibility. We can check that, by the above compatibility of $\left(\mathbb{M}_{G}, E\right)$ and $\left(\mathbb{M}_{G}, \tau\right)$, the $\mathbb{D}_{G}$-valued free distribution $\sigma_{x_{1}, \ldots, x_{s}}$ and the scalar-valued free distribution $\nu_{x_{1}, \ldots, x_{s}}$ of arbitrarily chosen operators $x_{1}, \ldots, x_{s}$ in $\mathbb{M}_{G}$ have similar data up to $\mathbb{D}_{G}$. However, even though the pairs $\left(\mathbb{M}_{G}, E\right)$ and $\left(\mathbb{M}_{G}, \tau\right)$ are compatible, the scalar-valued freeness on $\mathbb{M}_{G}$ (with respect to $\tau$ ) is not related the $\mathbb{D}_{G}$-valued reduced freeness on $\mathbb{M}_{G}$ (with respect to $E)$.

We consider the special case where $M=\mathbb{C}$. In this case, the corresponding graph von Neumann algebra $\mathbb{M}_{G}=\mathbb{C} \times_{\alpha} \mathbb{G}$ is always $*$-isomorphic to the groupoid $W^{*}$-algebra $\overline{\mathbb{C}[\mathbb{G}]}{ }^{w}$, generated by the (graph) groupoid $\mathbb{G}$ for all 
groupoid actions $\alpha$, by the linearity of $\alpha$ on $\mathbb{C}$. We show that, if $M=\mathbb{C}$, then the diagonal graph $W^{*}$-probability space $\left(\mathbb{M}_{G}, \tau\right)$ is always tracial.

\section{Preliminaries}

In this section, we introduce some basic concepts we need in the context and we review the fundamental results about graph von Neumann algebras.

\subsection{Free probability}

Let $B \subset A$ be von Neumann algebras with $1_{B}=1_{A}$ and assume that there is a conditional expectation $E_{B}: A \rightarrow B$ satisfying that (i) $E_{B}$ is a $\mathbb{C}$-linear map, (ii) $E_{B}(b)=b$ for all $b \in B$, (iii) $E_{B}\left(b_{1} a b_{2}\right)=b_{1} E_{B}(a) b_{2}$ for all $b_{1}, b_{2} \in$ $B$ and $a \in A$, (iv) $E_{B}$ is continuous under the given topology, and (v) $E_{B}\left(a^{*}\right)$ $=E_{B}(a)^{*}$ for all $a \in A$. The algebraic pair $\left(A, E_{B}\right)$ is said to be a $B$-valued $W^{*}$-probability space (over $B$ ). Every operator in $\left(A, E_{B}\right)$ is called a $B$-valued random variable. Any arbitrary chosen $B$-valued random variables have their free distributional data called $B$-valued $*$-moments and $B$-valued $*$-cumulants. Suppose $a_{1}, \ldots, a_{s}$ are $B$-valued random variables in $\left(A, E_{B}\right)$, where $s \in \mathbb{N}$. The $\left(i_{1}, \ldots, i_{n}\right)$-th joint $B$-valued $*$-moments of $a_{1}, \ldots, a_{s}$ are defined by

$$
E_{B}\left(a_{i_{1}}^{r_{i_{1}}} a_{i_{2}}^{r_{i_{2}}} \ldots a_{i_{n}}^{r_{i_{n}}}\right),
$$

and the $\left(j_{1}, \ldots, j_{k}\right)$-th joint $B$-valued $*$-cumulants of $a_{1}, \ldots, a_{s}$ are defined by

$$
k_{k}^{B}\left(a_{j_{1}}^{r_{i_{1}}}, a_{j_{2}}^{r_{j_{2}}}, \ldots, a_{j_{k}}^{r_{j_{k}}}\right)=\sum_{\pi \in N C(k)} E_{B: \pi}\left(a_{j_{1}}^{r_{j_{1}}}, \ldots, a_{j_{k}}^{r_{j_{k}}}\right) \mu\left(\pi, 1_{n}\right)
$$

for all $\left(i_{1}, \ldots, i_{n}\right) \in\{1, \ldots, s\}^{n}$ and for all $\left(j_{1}, \ldots, j_{k}\right) \in\{1, \ldots, s\}^{k}$, where $r_{i_{1}}, \ldots, r_{i_{n}}, r_{j_{1}}, \ldots, r_{j_{k}} \in\{1, *\}$ and $N C(n)$ is the lattice of all noncrossing partitions with its minimal element $0_{n}=\{(1),(2), \ldots,(n)\}$ and its maximal element $1_{n}=\{(1,2, \ldots, n)\}$, and $\mu$ is the Möbius functional in the incidence algebra satisfying that $\sum_{\pi \in N C(n)} \mu\left(\pi, 1_{n}\right)=0$. Here $E_{B: \pi}(\cdots)$ is the partitiondepending $B$-valued moment. For example, if $\pi=\{(1,4),(2,3),(5)\}$ in $N C(5)$, then

$$
E_{B: \pi}\left(a_{1}, a_{2}, a_{3}, a_{4}, a_{5}\right)=E_{B}\left(a_{1} E_{B}\left(a_{2} a_{3}\right) a_{4}\right) E_{B}\left(a_{5}\right) .
$$

Recall that the set $N C(n)$ of all noncrossing partitions over $\{1, \ldots, n\}$ is a lattice under the following ordering;

$$
\pi \leq \theta \stackrel{\text { def }}{\Longleftrightarrow} \forall V \in \pi, \exists B \in \theta \text { such that } V \subseteq B,
$$

where " $\subseteq$ " is the usual set-inclusion.

The $B$-valued freeness on $\left(A, E_{B}\right)$ is characterized by the $B$-valued cumulants (See [13]). Let $A_{1}$ and $A_{2}$ be $W^{*}$-subalgebras of $A$ having their common subalgebra $B$. We say that $A_{1}$ and $A_{2}$ are free over $B$ in $\left(A, E_{B}\right)$ if all mixed $B$-valued *-cumulants of $A_{1}$ and $A_{2}$ vanish. The subsets $X_{1}$ and $X_{2}$ of $A$ are said to be free over $B$ in $\left(A, E_{B}\right)$ if the von Neumann algebras $v N\left(X_{1}, B\right)$ and 
$v N\left(X_{2}, B\right)$ are free over $B$ in $\left(A, E_{B}\right)$, where $v N\left(S_{1}, S_{2}\right)$ means the von Neumann algebra generated by sets $S_{1}$ and $S_{2}$. Similarly, we say that the $B$-valued random variables $x$ and $y$ are free over $B$ in $\left(A, E_{B}\right)$ if the subsets $\{x\}$ and $\{y\}$ are free over $B$.

Let $A$ and $B$ be given as above, and assume that $A_{1}$ and $A_{2}$ are $W^{*}$ subalgebras of $A$, containing $B$. Then we can create a new $W^{*}$-subalgebra $A_{0}=v N\left(A_{1}, A_{2}\right)$ of $A$ (containing $B$ ). If $A_{1}$ and $A_{2}$ are free over $B$ in $\left(A, E_{B}\right)$, then the $W^{*}$-algebra $A_{0}$ is denoted by $A_{1} *_{B} A_{2}$, and it is called the $B$-valued free product algebra of $A_{1}$ and $A_{2}$. Similarly, assume that $\left\{A_{i}: i \in I\right\}$ is the family of $W^{*}$-subalgebras of $A$ containing $B$, and assume that $A_{i}$ 's are free over $B$ in $\left(A, E_{B}\right)$, from each other, and suppose they generate $A$, i.e., $A=v N\left(\left\{A_{i}: i \in I\right\}\right)$. Then $A$ is $*$-isomorphic to the $B$-valued free product ${ }^{*}{ }_{B} A_{i}$ of $A_{i}$ 's. In this case, we call $A$, a $B$-valued free product algebra of $i \in I$ $\left\{A_{i}: i \in I\right\}$.

\subsection{Graph groupoids and graph von Neumann algebras}

Let $G=(V(G), E(G), s, r)$ be a countable directed graph with its vertex set $V(G)$, its edge set $E(G)$, and its source and range maps $s$ and $r$. The maps $s, r: E(G) \rightarrow V(G)$ are determined by the direction on $G$;

$$
s(e) \stackrel{\text { def }}{=} \text { the initial vertex of } e
$$

and

$$
r(e) \stackrel{\text { def }}{=} \text { the terminal vertex of } e
$$

for all $e \in E(G)$. Let $e \in E(G)$ be an edge with $s(e)=v$ and $r(e)=v^{\prime}$ in $V(G)$. Then we write $e$ by $e=v e v^{\prime}$. Notice that $v$ and $v^{\prime}$ are not necessarily distinct in $V(G)$. For instance, if $e$ is a loop edge, then $v=v^{\prime}$.

Suppose $e_{1}$ and $e_{2}$ are edges in $E(G)$, and assume that they are connected in the order $e_{1} \rightarrow e_{2}$. Then we can determine the finite path generated by $e_{1}$ and $e_{2}$ on $G$, denoted by $e_{1} e_{2}$. More generally, if the edges $e_{1}, \ldots, e_{k}$ are connected in the order $e_{1} \rightarrow e_{2} \rightarrow \cdots \rightarrow e_{k}$ for $k \in \mathbb{N}$, then we can have the finite path $e_{1} e_{2} \cdots e_{k}$, generated by the edges. Denote the set of all finite paths of $G$ by $F P(G)$. Notice that all elements in $F P(G)$ are words in $E(G)$, dependent upon the direction on $G$. Clearly, the edge set $E(G)$ is contained in $F P(G)$.

If there is a finite path $w=e_{1} \cdots e_{n}$ in $F P(G)$, generated by the edges $e_{1}, \ldots, e_{n} \in E(G)$ for $n \in \mathbb{N}$, then we say that the directed edges $e_{1}, \ldots, e_{n}$ are admissible (or connected in the order $\left(e_{1}, \ldots, e_{n}\right)$ ). If $w$ is given as before, then the length $|w|$ of $w$ is defined to be $n$, which is the cardinality of the admissible edges generating $w$. Also, we say that finite paths $w_{1}=e_{11} \cdots e_{1 k_{1}}$ and $w_{2}=e_{21} \cdots e_{2 k_{2}}$ are admissible (or connected in the order $\left(w_{1}, w_{2}\right)$ ), if $w_{1}$ $w_{2}=e_{11} \cdots e_{1 k_{1}} e_{21} \cdots e_{2 k_{2}}$ is again an element of $F P(G)$, where $e_{11}, \ldots, e_{1 k_{1}}$, $e_{21}, \ldots, e_{2 k_{2}} \in E(G)$. Otherwise, we say that $w_{1}$ and $w_{2}$ are not admissible. By definition, $\left|w_{1} w_{2}\right|=\left|w_{1}\right|+\left|w_{2}\right|$, whenever $w_{1}$ and $w_{2}$ are admissible in $F P(G)$ for $w_{1}, w_{2} \in F P(G)$. 
Suppose that $w$ is a finite path in $F P(G)$ with its initial vertex $v_{1}$ and its terminal vertex $v_{2}$. We write $w=v_{1} w$ or $w=w v_{2}$ or $w=v_{1} w v_{2}$, for emphasizing the initial vertex of $w$, respectively, the terminal vertex of $w$, respectively, both the initial vertex and the terminal vertex of $w$. Suppose $w$ $=v_{1} w v_{2}$ in $F P(G)$ with $v_{1}, v_{2} \in V(G)$. Then we say that " $v_{1}$ and $w$ are admissible" and " $w$ and $v_{2}$ are admissible". Notice that even though finite paths $w_{1}$ and $w_{2}$ are admissible, $w_{2}$ and $w_{1}$ are not admissible, in general. For instance, if $e_{1}=v_{1} e_{1} v_{2}$ is an edge with $v_{1} \neq v_{2}$ in $V(G)$ and $e_{2}=v_{2} e_{2} v_{2}$ is a loop-edge in $E(G)$, then there is a finite path $e_{1} e_{2}$ in $F P(G)$, but there is no (nonempty) finite path $e_{2} e_{1}$ in $F P(G)$.

The free semigroupoid $\mathbb{F}^{+}(G)$ of $G$ is defined by a set

$$
\mathbb{F}^{+}(G)=\{\emptyset\} \cup V(G) \cup F P(G),
$$

with its binary operation $(\cdot)$ on $\mathbb{F}^{+}(G)$, defined by

$$
\left(w_{1}, w_{2}\right) \longmapsto w_{1} \cdot w_{2}= \begin{cases}w_{1} & \text { if } w_{1}=w_{2} \text { in } V(G), \\ w_{1} & \text { if } w_{1} \in F P(G), w_{2} \in V(G) \text { and } w_{1}=w_{1} w_{2}, \\ w_{2} & \text { if } w_{1} \in V(G), w_{2} \in F P(G) \text { and } w_{2}=w_{1} w_{2}, \\ w_{1} w_{2} & \text { if } w_{1}, w_{2} \text { in } F P(G) \text { and } w_{1} w_{2} \in F P(G), \\ \emptyset & \text { otherwise, }\end{cases}
$$

where $\emptyset$ is the empty word in $V(G) \cup E(G)$. The binary operation on $\mathbb{F}^{+}(G)$ is called the admissibility, i.e., the pair $\left(\mathbb{F}^{+}(G), \cdot\right)$ is the free semigroupoid of $G$. For convenience, we denote the free semigroupoid of $G$ by $\mathbb{F}^{+}(G)$.

For the given countable directed graph $G$, we can define a new countable directed graph $G^{-1}$, by the opposite directed graph of $G$, with

$$
V\left(G^{-1}\right)=V(G) \text { and } E\left(G^{-1}\right)=\left\{e^{-1}: e \in E(G)\right\},
$$

where $e^{-1} \in E\left(G^{-1}\right)$ is the opposite directed edge of $e \in E(G)$, i.e., if $e=$ $v_{1} e v_{2}$ with $v_{1}, v_{2} \in V(G)$, then $e^{-1}=v_{2} e^{-1} v_{1}$ with $v_{2}, v_{1} \in V\left(G^{-1}\right)$ $=V(G)$. The graph $G^{-1}$ is called the shadow of $G$. Similar to the previous paragraph, we can construct the set $F P\left(G^{-1}\right)$ of all finite paths of $G^{-1}$ and the free semigroupoid $\mathbb{F}^{+}\left(G^{-1}\right)$ of $G^{-1}$. The admissibility on $\mathbb{F}^{+}\left(G^{-1}\right)$ is oppositely preserved by that on $\mathbb{F}^{+}(G)$, since

$$
\left(G^{-1}\right)^{-1}=G
$$

In other words, if there is a finite path $w$ in $\mathbb{F}^{+}(G)$, then there is the corresponding shadow $w^{-1}$ in $\mathbb{F}^{+}\left(G^{-1}\right)$, and vice versa;

$$
w=e_{1} \cdots e_{n} \in F P(G) \Longleftrightarrow w^{-1}=e_{n}^{-1} \cdots e_{1}^{-1} \in F P\left(G^{-1}\right),
$$

where $e_{1}, \ldots, e_{n} \in E(G)$.

A new countable directed graph $G^{\wedge}$ is called the shadowed graph of $G$, if it is a directed graph with its vertex set

$$
V\left(G^{\wedge}\right)=V(G)=V\left(G^{-1}\right)
$$


and its edge set

$$
E\left(G^{\wedge}\right)=E(G) \cup E\left(G^{-1}\right) .
$$

Notice that, in general,

$$
\mathbb{F}^{+}\left(G^{\wedge}\right) \supset\left(\mathbb{F}^{+}(G) \cup \mathbb{F}^{+}\left(G^{-1}\right)\right) .
$$

Definition 2.1. Let $G$ be a countable directed graph and $\hat{G}$, the shadowed graph of $G$, and let $\mathbb{F}^{+}\left(G^{\wedge}\right)$ be the free semigroupoid of $G^{\wedge}$. Define the reduction $(\mathrm{RR})$ on $\mathbb{F}^{+}\left(G^{\wedge}\right)$ by

$$
w w^{-1}=v \text { and } w^{-1} w=v^{\prime},
$$

whenever $w=v w v^{\prime}$ in $F P\left(G^{\wedge}\right)$ with $v, v^{\prime} \in V\left(G^{\wedge}\right)$. The set $\mathbb{F}^{+}\left(G^{\wedge}\right)$, with this reduction $(\mathrm{RR})$, is denoted by $\mathbb{F}_{r}^{+}\left(G^{\wedge}\right)$ and this set with inherited admissibility from $\mathbb{F}^{+}\left(G^{\wedge}\right)$ is called a graph groupoid of $G$. Denote this graph groupoid $\left(\mathbb{F}_{r}^{+}\left(G^{\wedge}\right), \cdot\right)$ of $G$ by $\mathbb{G}$.

Now, construct the graph Hilbert space $H_{G}$, induced by the given graph $G$.

Definition 2.2. Let $G$ be the given countable directed graph with its graph groupoid $\mathbb{G}$. Define the Hilbert space $H_{G}$ by

$$
H_{G} \stackrel{\text { def }}{=} \underset{w \in F P_{r}\left(G^{\wedge}\right)}{\oplus}\left(\mathbb{C} \xi_{w}\right)
$$

with its Hilbert basis $\mathcal{B}_{H_{G}}=\left\{\xi_{w}: w \in F P_{r}\left(G^{\wedge}\right)\right\}$, where

$$
F P_{r}(\hat{G)} \stackrel{\text { def }}{=} \mathbb{G} \backslash(V(\hat{G}) \cup\{\emptyset\}) \text {. }
$$

Notice that the graph Hilbert space $H_{G}$ is in fact isomorphic to the Hilbert space $\underset{w \in F P_{r}\left(G^{\wedge}\right)}{\oplus} K_{G} \cdot \xi_{w}$, where $K_{G}$ is a Hilbert space isomorphic to

$$
\underset{v \in V\left(G^{\wedge}\right)}{\oplus}\left(\mathbb{C} \xi_{v}\right) .
$$

Since $\xi_{w}=\xi_{v w}=\xi_{v} \xi_{w}$, the direct summand $K_{G} \cdot \xi_{w}$ is isomorphic to $\mathbb{C} \xi_{w}$ for all $w \in F P_{r}\left(G^{\wedge}\right)$. Remark that if $w=e_{1} \cdots e_{k}$ is in $F P_{r}\left(G^{\wedge}\right)$, then $\xi_{w}$ can be regarded as $\xi_{e_{1}} \otimes \cdots \otimes \xi_{e_{k}}$, i.e., the summand $\mathbb{C} \xi_{w}$ is isomorphic to $\mathbb{C} \xi_{e_{1}} \otimes \cdots \otimes \mathbb{C} \xi_{e_{k}}$.

On $H_{G}$, we have the following multiplication rule;

$$
\xi_{w_{1}} \xi_{w_{2}}= \begin{cases}\xi_{w_{1} w_{2}} & \text { if } w_{1} w_{2} \neq \emptyset \\ \xi_{\emptyset} \stackrel{\text { def }}{=} 0_{H_{G}} & \text { otherwise }\end{cases}
$$

for all $w_{1}, w_{2} \in F P_{r}\left(G^{\wedge}\right)$, by the admissibility on $\mathbb{G}$. Notice that the product $w_{1} w_{2}$ of $w_{1}$ and $w_{2}$ means the reduced word gotten from the product $w_{1} \cdot w_{2}$ in $\mathbb{G}$. For instance, if $w_{1}=e_{1} e_{2}$ and $w_{2}=e_{2}^{-1}$, then the product $w_{1} w_{2}$ means $e_{1}$ in $\mathbb{G}$.

By the above multiplication rule on $\mathcal{B}_{H_{G}}$, if $\xi_{w} \in \mathcal{B}_{H_{G}}$, then

$$
\xi_{w} \xi_{w^{-1}}=\xi_{w w^{-1}}
$$


and $w w^{-1}$ is the initial vertex of $w \in F P_{r}\left(G^{\wedge}\right)$. This shows that there exist Hilbert space elements determined by vertices. We will denote them by $\xi_{v}$ in $H_{G}$ for all $v \in V\left(G^{\wedge}\right)$. Thus, for any $w \in \mathbb{G}$, we can have the corresponding Hilbert space element $\xi_{w}$ in $H_{G}$. Moreover, by the admissibility on $\mathbb{G}$, for any $w_{1}, w_{2} \in \mathbb{G}$, we can have the same multiplication rule as above.

The above multiplication rule on $H_{G}$ guarantees the existence of multiplication operators on $H_{G}$.

Definition 2.3. An operator $L_{w}$ is defined by the multiplication operator with its symbol $\xi_{w}$ on $H_{G}$ for $w \in \mathbb{G}$, i.e.,

$$
L_{w} \xi_{w^{\prime}}= \begin{cases}\xi_{w} \xi_{w^{\prime}}=\xi_{w w^{\prime}} & \text { if } w w^{\prime} \neq \emptyset \\ \xi_{\emptyset}=0_{H_{G}} & \text { otherwise }\end{cases}
$$

for all $w, w^{\prime} \in \mathbb{G}$. The adjoint $L_{w}^{*}$ of $L_{w}$ is defined by $L_{w}^{*}=L_{w^{-1}}$ for all $w \in$ $\mathbb{G}$. Then

Let $L_{w_{1}}$ and $L_{w_{2}}$ be the multiplication operators on $H_{G}$, where $w_{1}, w_{2} \in \mathbb{G}$.

$$
L_{w_{1}} L_{w_{2}}=L_{w_{1} w_{2}} \text { on } H_{G} \text {. }
$$

Therefore, the multiplication operators $L_{v}$ 's are projections for all $v \in$ $V\left(G^{\wedge}\right)$. Indeed, we have that

$$
L_{v}^{*}=L_{v^{-1}}=L_{v}=L_{v^{2}}=L_{v}^{2}
$$

for all $v \in V\left(G^{\wedge}\right)$. And the multiplication operators $L_{w}$ 's and $L_{w}^{*}$ 's are partial isometries for all $w \in F P_{r}\left(G^{\wedge}\right)$, since

$$
L_{w} L_{w}^{*}=L_{w} L_{w^{-1}}=L_{w w^{-1}} \text { and } L_{w}^{*} L_{w}=L_{w^{-1}} L_{w}=L_{w^{-1} w} .
$$

We need one more ingredient to construct our graph von Neumann algebra.

Definition 2.4. Let $\mathbb{G}$ be a graph groupoid of a countable directed graph $G$ and let $M$ be a von Neumann algebra. Define a groupoid action $\alpha$ of $\mathbb{G}$, acting on $M$, by a nonunital partial representation satisfying that

$$
\alpha_{w}(m) L_{w} L_{w}^{*}=L_{w}^{*} m L_{w}=L_{w^{-1}} m L_{w}
$$

for all $m \in M$ and $w \in \mathbb{G}$, where $L_{w}$ is a multiplication operator on $H_{G}$. We can regard $L_{w}$ as elements $1_{M} \otimes L_{w}$ in $B\left(K \otimes H_{G}\right)$ for all $w \in \mathbb{G}$. Also, define

$$
\alpha_{v}(m)=m \text { for all } m \in M \text { and } v \in V\left(G^{\wedge}\right) .
$$

Notation. For convenience, denote $\alpha_{w}(m)$ by $m^{w}$ for $w \in \mathbb{G}$ and $m \in M$.

Definition 2.5. Define the crossed product $\mathbb{M}_{G}=M \times_{\alpha} \mathbb{G}$ of $M$ and $\mathbb{G}$, via a groupoid action $\alpha$, by the von Neumann algebra generated by $M$ and $\left\{L_{w}\right.$ : $w \in \mathbb{G}\}$ in $B\left(K \otimes H_{G}\right)$, satisfying (2.1) and $(2.1)^{\prime}$. This von Neumann algebra $\mathbb{M}_{G}$ is called a graph von Neumann algebra generated by $G$ over $M$. 


\subsection{Amalgamated free probability on graph von Neumann algebras}

Let $G$ be a countable directed graph with its graph groupoid $\mathbb{G}$. Every operator $x$ in a graph von Neumann algebra $\mathbb{M}_{G}=M \times_{\alpha} \mathbb{G}$ has its expression,

$$
x=\sum_{w \in \mathbb{G}} m_{w} L_{w} \text { for } m_{w} \in M .
$$

A graph von Neumann algebra $\mathbb{M}_{G}$ always has its $W^{*}$-subalgebra $\mathbb{D}_{G}$ defined by

$$
\mathbb{D}_{G} \stackrel{\text { def }}{=} \underset{v \in V\left(G^{\wedge}\right)}{\bigoplus}\left(M \cdot L_{v}\right)
$$

Notice that this $W^{*}$-subalgebra $\mathbb{D}_{G}$ is in fact determined without the choice of a groupoid action $\alpha$, i.e., even if $\alpha_{1}$ and $\alpha_{2}$ are distinct groupoid actions of $\mathbb{G}$ acting on $M$, the $W^{*}$-subalgebra $\mathbb{D}_{G}$ is uniquely determined in both $M \times_{\alpha_{1}}$ $\mathbb{G}$ and $M \times_{\alpha_{2}} \mathbb{G}$. So, we call this $W^{*}$-subalgebra of $\mathbb{M}_{G}$, "the" $M$-diagonal subalgebra of $\mathbb{M}_{G}$.

When we have the $W^{*}$-subalgebra inclusion $\mathbb{D}_{G} \subset \mathbb{M}_{G}$, we can construct the canonical conditional expectation $E: \mathbb{M}_{G} \rightarrow \mathbb{D}_{G}$ by

$$
E\left(\sum_{w \in \mathbb{G}} m_{w} L_{w}\right)=\sum_{v \in V\left(G^{\wedge}\right)} m_{v} L_{v} .
$$

Then the pair $\left(\mathbb{M}_{G}, E\right)$ is an amalgamated $W^{*}$-probability space over $\mathbb{D}_{G}$. This $\mathbb{D}_{G^{\text {-valued }}} W^{*}$-probability space $\left(\mathbb{M}_{G}, E\right)$ is called a $M$-diagonal graph $W^{*}$-probability space over $\mathbb{D}_{G}$.

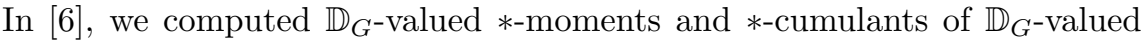
random variables $m_{1} L_{w_{1}}, \ldots, m_{n} L_{w_{n}}$ in the $M$-diagonal graph $W^{*}$-probability space $\left(\mathbb{M}_{G}, E\right)$ for $m_{1}, \ldots, m_{n} \in M$, and $w_{1}, \ldots, w_{n} \in \mathbb{G}$ for $n \in \mathbb{N}$;

Proposition 2.1 $([6])$. Let $\left(\mathbb{M}_{G}=M \times_{\alpha} \mathbb{G}, E\right)$ be a $M$-diagonal graph $W^{*}$ probability space over its $M$-diagonal subalgebra $\mathbb{D}_{G}$, and let $m_{1} L_{w_{1}}, \ldots, m_{n} L_{w_{n}}$ be $\mathbb{D}_{G}$-valued random variables for $n \in \mathbb{N}$. Then

$$
\begin{aligned}
E\left(m_{1} L_{w_{1}} \cdots m_{n} L_{w_{n}}\right) & =m_{0} E\left(L_{w_{1} \cdots w_{n}}\right), \\
k_{n}\left(m_{1} L_{w_{1}}, \ldots, m_{n} L_{w_{n}}\right) & =\left(\mu_{0} \cdot m_{0}\right) E\left(L_{w_{1} \cdots w_{n}}\right),
\end{aligned}
$$

where

$$
m_{0} \stackrel{\text { def }}{=} m_{1} m_{2}^{w_{1}} m_{3}^{\left(w_{1} w_{2}\right)^{-1}} \cdots m_{n}^{\left(w_{1} \cdots w_{n-1}\right)^{-1}} \in M
$$

and

$$
\mu_{0} \stackrel{\text { def }}{=} \sum_{\pi \in N C\left(w_{1}, \ldots, w_{n}\right)} \mu\left(\pi, 1_{n}\right) \in \mathbb{C},
$$

where $m^{w}$ means $\alpha_{w}(m)$ for all $m \in M$ and $w \in \mathbb{G}$ and where

$$
N C\left(w_{1}, \ldots, w_{n}\right) \stackrel{\text { def }}{=}\left\{\theta \in N C(n): E_{\theta}\left(L_{w_{1}}, \ldots, L_{w_{n}}\right)=E\left(L_{w_{1} \ldots w_{n}}\right)\right\} .
$$

Based on the above computations, we showed that: 
Theorem 2.2 (See [6]). The subsets $M \cdot L_{w_{1}}$ and $M \cdot L_{w_{2}}$ are free over $\mathbb{D}_{G}$ in $\left(\mathbb{M}_{G}, E\right)$ if and only if $w_{1}$ and $w_{2}$ are diagram-distinct in the sense that (i) $w_{1} \neq w_{2}^{-1}$ in $\mathbb{G}$, and (ii) the diagrams (or the graphical images) of $w_{1}$ and $w_{2}$ are distinct on $G$.

Let $X_{1}$ and $X_{2}$ be self-adjoint subsets of $\mathbb{G}$ in the sense that $X_{k}^{-1}=X_{k}$ for $k=1,2$. Then we define the reduced free product $X_{1} * X_{2}$ "inside" $\mathbb{G}$ by the collection of all reduced words in $X_{1} \cup X_{2}$, where the reduction is depending on the admissibility and (RR) on $\mathbb{G}$. Notice that if $X$ is a self-adjoint subset of $\mathbb{G}$ and if $\mathbb{X}$ is a collection of all reduced words in $X$ (as a subset of $\mathbb{G}$ ), then the set $\mathbb{X}$ is again a graph groupoid of a certain graph $X$, having the same admissibility with $\mathbb{G}$. So, we can create a $W^{*}$-subalgebra $M \times_{\alpha_{X}} \mathbb{X}$ in $\mathbb{M}_{G}$ which is a new graph von Neumann algebra, where $\alpha_{X}=\left.\alpha\right|_{\mathbb{X}}$. So, more general to the previous theorem, we can get the following theorem.

Theorem 2.3 (See [6]). If $X_{1}$ and $X_{2}$ are diagram-distinct self-adjoint subsets in $\mathbb{G}$, and if $X_{1} * X_{2}$ is a free product of $X_{1}$ and $X_{2}$ inside $\mathbb{G}$, then

$$
\mathbb{M}_{X_{1} * X_{2}}=\left(\mathbb{M}_{X_{1}}\right) * \mathbb{D}_{G}\left(\mathbb{M}_{X_{2}}\right),
$$

where $\mathbb{M}_{S} \stackrel{\text { def }}{=} v N\left(M \times_{\alpha_{S}} \mathbb{S}, \mathbb{D}_{G}\right)$ is a $W^{*}$-subalgebra of $\mathbb{M}_{G}$, where $M \times_{\alpha_{S}} \mathbb{S}$ is a certain graph von Neumann algebra induced by the graph $G_{S}$ and $\alpha_{S}=\left.\alpha\right|_{\mathbb{S}}$ is the groupoid action of $\mathbb{S}$.

As a corollary of the previous theorem, we can get the following theorem.

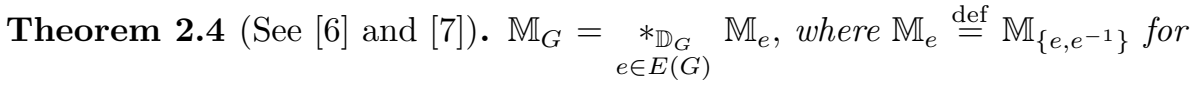
all $e \in E(G)$.

This shows that our graph von Neumann algebras are $*$-isomorphic to amalgamated (reduced) free product algebras. However, the above theorem does not explain the reduction of the $\mathbb{D}_{G}$-free product “ ${ }^{*} \mathbb{D}_{G}$ ", dependent upon the admissibility on $\mathbb{G}$.

Let $\left\{A_{i}: i \in I\right\}$ be a collection of $W^{*}$-subalgebras of $A$, containing their common $W^{*}$-subalgebra $B$. And assume that the von Neumann algebra $A$ is equipped with the conditional expectation $E_{B}: A \rightarrow B$. Also, suppose $A$ is *-isomorphic to a $B$-valued free product algebra $\underset{i \in I}{*_{B}} A_{i}$, with respect to $E_{B}$. Then $A$ has the following Banach-space expression,

$$
B \oplus\left(\oplus_{n=1}^{\infty}\left(\underset{i_{1} \neq i_{2}, i_{2} \neq i_{3}, \ldots, i_{n-1} \neq i_{n}}{\oplus}\left(A_{i_{1}}^{o} \otimes_{B} \cdots \otimes_{B} A_{i_{n}}^{o}\right)\right)\right),
$$

where $A_{i_{j}}^{o} \stackrel{\text { def }}{=} A_{i_{j}} \ominus B$ for all $j=1, \ldots, n$.

Since our graph von Neumann algebra $\mathbb{M}_{G}$ is $*$-isomorphic to a $\mathbb{D}_{G}$-free product algebra of $\mathbb{M}_{e}$ 's, it should have the similar Banach-space expression. However, it is checked that if all edges $\hat{e_{1}} \in\left\{e_{1}, e_{1}^{-1}\right\}$ and $\hat{e_{2}} \in\left\{e_{2}, e_{2}^{-1}\right\}$ are 
not admissible, then $\mathbb{M}_{e_{1}}^{o} \otimes_{\mathbb{D}_{G}} \mathbb{M}_{e_{2}}^{o}$ is a Banach-space isomorphic to the trivial vector space $\left\{0_{\mathbb{D}_{G}}\right\}$ (See $[6]$ ). Thus, we obtain the following theorem.

Theorem 2.5 (See [7]). The graph von Neumann algebra $\mathbb{M}_{G}$ is expressed as a Banach space,

$$
\mathbb{D}_{G} \oplus\left(\underset{w^{*} \in E(G)_{r}^{*}}{\oplus} \mathbb{M}_{w^{*}}^{o}\right)
$$

where

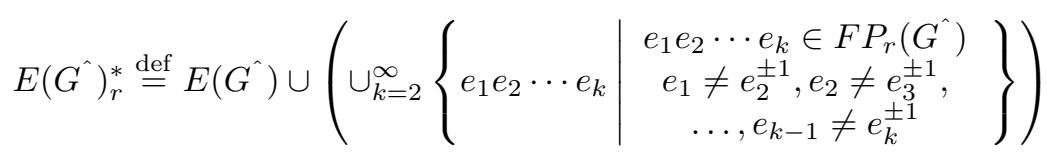

under the fact that $\mathbb{M}_{e}=\mathbb{M}_{e^{-1}}$ for all $e \in E(G)$, and

$$
\mathbb{M}_{w^{*}}^{o} \stackrel{\text { def }}{=} \mathbb{M}_{e_{1}}^{o} \otimes_{\mathbb{D}_{G}} \cdots \otimes_{\mathbb{D}_{G}} \mathbb{M}_{e_{n}}^{o}
$$

with

$$
\mathbb{M}_{e_{k}}^{o} \stackrel{\text { def }}{=} \mathbb{M}_{e_{k}} \ominus \mathbb{D}_{G},
$$

whenever $w^{*}=e_{1} \cdots e_{n}$ in $E\left(G^{\wedge}\right)_{r}^{*}$, with $e_{1}, \ldots, e_{n} \in E\left(G^{\wedge}\right)$.

The above theorem finally explain how the reduction of " ${ }_{\mathbb{D}_{G}}$ " works, i.e., it shows that the reduction is totally depending on a combinatorial data of the given graph $G$ (or the admissibility on $\mathbb{G}$ ).

Notation. To emphasize such reduction on $*_{\mathbb{D}_{G}}$, represented by the above theorem, we denote $*_{\mathbb{D}_{G}} \quad \mathbb{M}_{e}$ by $\quad *_{\mathbb{D}_{G}}^{r} \quad \mathbb{M}_{e}$.

$$
e \in E(G) \quad e \in E(G)
$$

It is easy to see that if a fixed von Neumann algebra $M$ is $\mathbb{C}$, then graph von Neumann algebras $\mathbb{C} \times_{\alpha} \mathbb{G}$ are all $*$-isomorphic to $\overline{\mathbb{C}[\mathbb{G}]}$ in $B\left(H_{G}\right)$ for all groupoid actions $\alpha$, by the linearity on $\mathbb{C}$, i.e., if $M=\mathbb{C}$, then the construction of graph von Neumann algebras $\mathbb{C} \times_{\alpha} \mathbb{G}$ is free from the choice of groupoid actions of $\mathbb{G}$. Equivalently, if $M=\mathbb{C}$, then our graph von Neumann algebras are nothing but groupoid $W^{*}$-algebras, generated by graph groupoids.

If $G_{e}$ is a directed graph with only one edge $e$, with its graph groupoid $\mathbb{G}_{e}$, then the graph von Neumann algebra $\overline{\mathbb{C}\left[\mathbb{G}_{e}\right]}$ is $*$-isomorphic to either $L^{\infty}(\mathbb{T})$ or $M_{2}(\mathbb{C})$, where $\mathbb{T}$ is a unit circle in $\mathbb{C}$ and $M_{2}(\mathbb{C})$ is the matricial algebra generated by all $(2 \times 2)$-matrices. In general, a graph von Neumann algebra $\mathbb{M}_{G_{e}}=M \times{ }_{\alpha} \mathbb{G}_{e}$ is $*$-isomorphic to either a classical crossed product algebra $M \times_{\gamma} \mathbb{Z}$ or a $W^{*}$-subalgebra $M_{2}^{\alpha}(M)$ of the von Neumann algebra $M_{2}(M)=$ $M \otimes_{\mathbb{C}} M_{2}(\mathbb{C})$.

Motivated by the previous examples, in [7], we proved that:

Theorem 2.6 (See [7]). Let $\mathbb{M}_{G}=M \times_{\alpha} \mathbb{G}$ be a graph von Neumann algebra, *-isomorphic to the $\mathbb{D}_{G}$-valued reduced free product algebra $*_{\mathbb{D}_{G}}^{r} \mathbb{M}_{e}$, with $\mathbb{D}_{G}$-free blocks $\left\{\mathbb{M}_{e}: e \in E(G)\right\}$. 
(1) If $e$ is a loop edge, then $\mathbb{M}_{e}=v N\left(M \times_{\lambda^{(e)}} \mathbb{Z}, \mathbb{D}_{G}\right)$, where the group action $\lambda^{(e)}$ is identified with $\left.\alpha\right|_{\mathbb{G}_{e}}$, where $\mathbb{G}_{e}$ is the subset of $\mathbb{G}$ consisting of all reduced words in $\left\{e, e^{-1}\right\}$.

(2) If $e$ is a non-loop edge, then $\mathbb{M}_{e}=v N\left(M_{2}^{\alpha_{e}}(M), \mathbb{D}_{G}\right)$, where

$$
M_{2}^{\alpha_{e}}(M) \stackrel{\text { def }}{=} v N\left(M,\left\{\left(\begin{array}{ll}
0 & 1 \\
0 & 0
\end{array}\right)\right\} \mid\left(\begin{array}{cc}
\alpha_{e}(m) & 0_{M} \\
0_{M} & 0_{M}
\end{array}\right)=\left(\begin{array}{cc}
0_{M} & 0_{M} \\
0_{M} & m \\
\forall m \in M
\end{array}\right),,\right.
$$

in $M_{2}(M)=M \otimes_{\mathbb{C}} M_{2}(\mathbb{C})$. In particular, if $M=\mathbb{C}$, then $\mathbb{M}_{e}$ is $*$-isomorphic to either $v N\left(L^{\infty}(\mathbb{T}), \mathbb{D}_{G}\right)$ or $v N\left(M_{2}(\mathbb{C}), \mathbb{D}_{G}\right)$.

We can see that the free group factor $L\left(F_{N}\right)$, generated by the free group $F_{N}$ with $N$-generators, is $*$-isomorphic to our graph von Neumann algebra $\overline{\mathbb{C}\left[\mathbb{G}_{N}\right]}{ }^{w}$, where $\mathbb{G}_{N}$ is the graph groupoid of the one-vertex- $N$-loop-edge graph $G_{N}$. By using the above $\mathbb{D}_{G}$-freeness, we can double-check the famous Voiculescu's theorem: $L\left(F_{N}\right)$ is $*$-isomorphic to $*_{j=1}^{N} L(\mathbb{Z})_{j}$, where $L(\mathbb{Z})_{j}=L(\mathbb{Z})$ for all $j=1, \ldots, N$, and hence, $L\left(F_{N}\right)$ is $*$-isomorphic to $L\left(F_{k_{1}}\right) * L\left(F_{k_{2}}\right)$, whenever $k_{1}+k_{2}=N$ in $\mathbb{N} \backslash\{1\}$ for $k_{1}, k_{2} \in \mathbb{N}$.

\subsection{Scalar-valued free probability on graph von Neumann algebras}

Now, let's observe the scalar-valued free probability on graph von Neumann algebras. To do that, we consider the general case first.

Let $\left(A, E_{B}\right)$ be a $B$-valued $W^{*}$-probability space with its conditional expectation $E_{B}: A \rightarrow B$. If $B=\mathbb{C}$ and the given conditional expectation $E_{B}$ is a continuous linear functional on $A$, then the $\mathbb{C}$-valued $W^{*}$-probability space $(A$, $\left.E_{B}\right)$ is called a ( $\mathbb{C}$-valued or scalar-valued) $W^{*}$-probability space. To emphasize $E_{B}$ is a linear functional, we denote $E_{B}$ by $\varphi$. It is easy to check that, in this case, $\varphi\left(a^{*}\right)=\varphi(a)^{*}=\overline{\varphi(a)}$ in $\mathbb{C}$ for all $a \in A$.

In particular, if $\varphi$ satisfies $\varphi\left(a_{1} a_{2}\right)=\varphi\left(a_{2} a_{1}\right)$ for all $a_{1}, a_{2} \in A$, we say that this linear functional $\varphi$ is a trace, and we call the pair $(A, \varphi)$, a tracial $W^{*}$-probability space. Similar to the amalgamated case, all elements $a$ in $(A, \operatorname{tr})$ are called (free) random variables. For any fixed random variables $a_{1}, \ldots, a_{s} \in$ $(A, \varphi)$ for $s \in \mathbb{N}$, they have their (scalar-valued or $\mathbb{C}$-valued) free distributional data:

$$
\left(i_{1}, \ldots, i_{n}\right) \text {-th joint } * \text {-moments }: \varphi\left(a_{i_{1}}^{r_{1}} \cdots a_{i_{n}}^{r_{n}}\right)
$$

and

$$
\left(j_{1}, \ldots, j_{m}\right) \text {-th joint } * \text {-cumulants }: k_{m}^{\varphi}\left(a_{j_{1}}^{r_{1}}, \ldots, a_{j_{m}}^{r_{m}}\right)
$$

for all $\left(i_{1}, \ldots, i_{n}\right) \in\{1, \ldots, s\}^{n}$ and $\left(j_{1}, \ldots, j_{m}\right) \in\{1, \ldots, s\}^{m}$ for all $n, m \in$ $\mathbb{N}$, and $r_{t} \in\{1, *\}$, where $k_{n}^{\varphi}(\cdots)$ is the ( $\mathbb{C}$-valued) cumulant determined by the given linear functional $\varphi$;

$$
k_{m}^{\varphi}\left(a_{j_{1}}^{r_{1}}, \ldots, a_{j_{n}}^{t_{m}}\right)=\sum_{\pi \in N C(m)} \varphi_{\pi}\left(a_{j_{1}}^{r_{1}}, \ldots, a_{j_{m}}^{r_{m}}\right) \mu\left(\pi, 1_{m}\right),
$$


by the Moebius inversion, where $\varphi_{\pi}(\cdots)$ means the partition-depending moments for all $\pi \in N C(m)$. But different from the amalgamated case, the above cumulant formula can be re-written by

$$
k_{m}^{\varphi}\left(a_{j_{1}}^{r_{1}}, \ldots, a_{j_{m}}^{r_{m}}\right)=\sum_{\pi \in N C(m)}\left(\prod_{V=\left(k_{1}, \ldots, k_{t}\right) \in \pi}\left(\varphi\left(a_{k_{1}}^{r_{k_{1}}} a_{k_{2}}^{r_{k_{2}}} \cdots a_{k_{t}}^{r_{k_{t}}}\right)\right)\right) \mu\left(\pi, 1_{m}\right),
$$

where " $V \in \pi$ " means that " $V$ is a block of $\pi$." The above computation is expressed by "there is no insertion property for computing cumulants" (See [30]). For instance, if $\pi=\{(1,4),(2,3),(5)\}$ in $N C(5)$, and if $a_{1}, \ldots, a_{5}$ are operators in $A$, then

$$
E_{B: \pi}\left(a_{1}, \ldots, a_{5}\right)=E_{B}\left(a_{1} E_{B}\left(a_{2} a_{3}\right) a_{4}\right) E_{B}\left(a_{5}\right) \text { in } B,
$$

whenever $E_{B}: A \rightarrow B$ is an arbitrary conditional expectation, where $B \subseteq A$. However, if $B=\mathbb{C}$, and if $E_{B}=\varphi$, then

$$
\begin{aligned}
\varphi_{\pi}\left(a_{1}, \ldots, a_{5}\right) & =\varphi\left(a_{1} \varphi\left(a_{2} a_{3}\right) a_{4}\right) \varphi\left(a_{5}\right) \\
& =\varphi\left(a_{1} a_{4}\right) \varphi\left(a_{2} a_{3}\right) \varphi\left(a_{5}\right),
\end{aligned}
$$

since all images of $\varphi$ are contained in $\mathbb{C}$, and $\mathbb{C}$ is commutative with all elements of $A$. So, more generally, we can conclude that: if $B=C_{A}(B)$, then there is no insertion property for computing cumulants, where

$$
C_{A}(B) \stackrel{\text { def }}{=}\{b \in B: a b=b a, \forall a \in A\} .
$$

Notice that, by the previous observation, we can easily verify that there is no relation between the $B$-valued freeness on $\left(A, E_{B}\right)$ and the freeness on $(A$, $\varphi)$, in general. However, it is possible there is a nice relationship between them under certain additional conditions.

Definition 2.6. Let $B \subseteq A$ be von Neumann algebras, and let $E_{B}: A \rightarrow B$ and $\varphi: A \rightarrow \mathbb{C}$ be a conditional expectation and a trace, respectively. We say that a $B$-valued $W^{*}$-probability space $\left(A, E_{B}\right)$ and a tracial $W^{*}$-probability space $(A, \varphi)$ are compatible if

$$
\varphi(a)=\varphi\left(E_{B}(a)\right) \text { for all } a \in A .
$$

Under the compatibility, the free distributional data with respect to $\varphi$ is depending on the amalgamated free distributional data with respect to $E_{B}$. Unfortunately, we can see that the freeness on $(A, \varphi)$ and the $B$-valued freeness on $\left(A, E_{B}\right)$ has no close connection even if they are compatible. However, we will show that, in our graph von Neumann algebraic case, such compatibility works nicely providing the similarity of the $\mathbb{D}_{G}$-valued freeness and the $\mathbb{C}$-freeness on a graph von Neumann algebra $\mathbb{M}_{G}$, with certain additional conditions. 


\section{Diagonal graph $W^{*}$-probability spaces}

Throughout this chapter, let $G$ be a finite directed graph. Recall that we say that a graph $G$ is finite, if $|V(G)|<\infty$ and $|E(G)|<\infty$. For an arbitrary fixed von Neumann algebra $M$ acting on a Hilbert space $K$, and for the fixed groupoid action $\alpha$ of $\mathbb{G}$ acting on $M$, in $B\left(K \otimes H_{G}\right)$, we construct a graph von Neumann algebra $\mathbb{M}_{G}=M \times_{\alpha} \mathbb{G}$, having its $M$-diagonal subalgebra $\mathbb{D}_{G}=$ $\underset{v \in V\left(G^{\wedge}\right)}{\oplus}\left(M \cdot L_{v}\right)$.

Let $\left(\mathbb{M}_{G}, E\right)$ be a $M$-diagonal graph $W^{*}$-probability space over $\mathbb{D}_{G}$. Assume now that the fixed von Neumann algebra $M$ is equipped with a trace $\operatorname{tr}: M$ $\rightarrow \mathbb{C}$, i.e., we have a tracial $W^{*}$-probability space $(M, \operatorname{tr})$. We say that a trace $\operatorname{tr}$ on $M$ is faithful, if it satisfies that; $\operatorname{tr}\left(m m^{*}\right)=0$ if and only if $m=0_{M}$.

Assumption. If we say the pair $(M, \operatorname{tr})$ is a tracial $W^{*}$-probability space, then the trace tr on $M$ is automatically assumed to be faithful.

The pair $(M, \operatorname{tr})$ is a tracial $W^{*}$-probability space. The direct summands $M \cdot L_{v}$ of the $M$-diagonal subalgebra $\mathbb{D}_{G}$ are all $*$-isomorphic to $M$ for all $v \in V\left(G^{\wedge}\right)$, and hence we can construct the tracial $W^{*}$-probability spaces $\left(M \cdot L_{v}, \operatorname{tr}_{v}\right)$, by defining the trace

$$
\operatorname{tr}_{v}: M \cdot L_{v} \rightarrow \mathbb{C} \cdot L_{v}=\mathbb{C},
$$

by

$$
\operatorname{tr}_{v}\left(m L_{v}\right) \stackrel{\text { def }}{=} \operatorname{tr}(m) \cdot L_{v} \text { for all } m L_{v} \in M \cdot L_{v}
$$

for all $v \in V\left(G^{\wedge}\right)$. Then we can have the direct producted amalgamated $W^{*}$ probability space $\left(\mathbb{D}_{G}, T\right)$, in the sense of [4], where

$$
T: \mathbb{D}_{G} \rightarrow D_{G} \stackrel{\text { def }}{=} \underset{v \in V\left(G^{\wedge}\right)}{\oplus}\left(\mathbb{C} \cdot L_{v}\right)
$$

defined by

$$
T \stackrel{\text { def }}{=} \underset{v \in V\left(G^{\wedge}\right)}{\oplus} \operatorname{tr}_{v}
$$

Remark that the von Neumann algebra $D_{G}$ is a well-determined $W^{*}$-subalgebra of the $M$-diagonal subalgebra $\mathbb{D}_{G}$. Moreover, by the finiteness of $G$,

$$
\mathbb{D}_{G} \stackrel{\text { *-isomorphic }}{=} M^{\oplus|V(G)|} \text { and } D_{G} \stackrel{\text { *-isomorphic }}{=} \mathbb{C}^{\oplus|V(G)|},
$$

and $D_{G}$ is embedded in $\mathbb{D}_{G}$. Therefore, the conditional expectation $T$ is welldefined. And by [4], since $\operatorname{tr}_{v}$ are traces, this conditional expectation is a trace, too, in the sense that $T\left(d_{1} d_{2}\right)=T\left(d_{2} d_{1}\right)$ for all $d_{1}, d_{2} \in \mathbb{D}_{G}$.

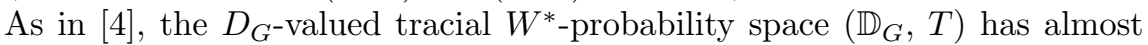
same free probabilistic properties with those of tracial $W^{*}$-probability space $(M, \operatorname{tr})$. Roughly speaking, we can understand the free probabilistic properties of $\left(\mathbb{D}_{G}, T\right)$ as the direct sum of the free probabilistic properties of $(M, \operatorname{tr})$. For example, if $W^{*}$-subalgebras $M_{1}$ and $M_{2}$ of $M$ are free in $(M, \operatorname{tr})$, then the $W^{*}$-subalgebras $\underset{v \in V\left(G^{\wedge}\right)}{\oplus} M_{1: v}$ and $\underset{v \in V\left(G^{\wedge}\right)}{\oplus} M_{2: v}$ are free over $D_{G}$ in $\left(\mathbb{D}_{G}, T\right)$, 
where $M_{k: v}=M_{k}$ or $\mathbb{C}$ for $k=1,2$ for $v \in V\left(G^{\wedge}\right)$, i.e., all free probabilistic data on $(M, \operatorname{tr})$ can be extendable to the $D_{G}$-valued free probabilistic data on $\left(\mathbb{D}_{G}, T\right)$, via direct product.

Now, define a trace $\operatorname{Tr}: \mathbb{D}_{G} \rightarrow \mathbb{C}$ by

$$
\operatorname{Tr} \stackrel{\text { def }}{=} \operatorname{tr}_{\left|V\left(G^{\wedge}\right)\right|} \circ T,
$$

where

$$
\operatorname{Tr}_{\left|V\left(G^{\wedge}\right)\right|}: D_{G} \rightarrow \mathbb{C}
$$

is a usual trace on $D_{G}$ (by regarding $D_{G}$ as the "diagonal" matricial algebra generated by all diagonal matrices in the matricial algebra $M_{\left|V\left(G^{\wedge}\right)\right|}(\mathbb{C})$ ) defined by

$$
\operatorname{Tr}_{\left|V\left(G^{\wedge}\right)\right|}\left(\underset{v \in V\left(G^{\wedge}\right)}{\oplus} t_{v}\right) \stackrel{\text { def }}{=} \sum_{v \in V\left(G^{\wedge}\right)} t_{v} \text { for all } t_{v} \in \mathbb{C} .
$$

Then the pair $\left(\mathbb{D}_{G}, \operatorname{tr}\right)$ is a "tracial" $W^{*}$-probability space. In conclusion, we can define a trace $\operatorname{Tr}$ on $\mathbb{D}_{G}$ by

$$
\operatorname{Tr}\left(\underset{v \in V\left(G^{\wedge}\right)}{\oplus} m_{v} L_{v}\right)=\sum_{v \in V\left(G^{\wedge}\right)} \operatorname{tr}_{v}\left(m_{v} L_{v}\right)=\sum_{v \in V\left(G^{\wedge}\right)} \operatorname{tr}\left(m_{v}\right) .
$$

Definition 3.1. The pair $\left(\mathbb{D}_{G}\right.$, tr) is called the diagonal tracial $W^{*}$-probability space.

Now, let's consider the graph von Neumann algebra $\mathbb{M}_{G}=M \times_{\alpha} \mathbb{G}$ over the diagonal tracial $W^{*}$-probability space $\left(\mathbb{D}_{G}, \operatorname{tr}\right)$.

Definition 3.2. Let $\left(\mathbb{M}_{G}, E\right)$ be a $M$-diagonal graph $W^{*}$-probability space and let $\left(\mathbb{D}_{G}, \operatorname{tr}\right)$ be the diagonal tracial $W^{*}$-probability space. Define a linear functional $\tau$ on $\mathbb{M}_{G}$ by

$$
\tau \stackrel{\text { def }}{=} \operatorname{tr} \circ E .
$$

The pair $\left(\mathbb{M}_{G}, \tau\right)$ is called the diagonal graph $W^{*}$-probability space.

Let $a_{k}=\sum_{w_{k} \in \mathbb{G}} m_{w_{k}}^{(k)} L_{w_{k}}$ be operators in $\mathbb{M}_{G}$ for $k=1,2$. Then

$$
\begin{aligned}
\tau\left(a_{1} a_{2}\right) & =\tau\left(\sum_{\left(w_{1}, w_{2}\right) \in \mathbb{G}^{2}}\left(m_{w_{1}}^{(1)} L_{w_{1}}\right)\left(m_{w_{2}}^{(2)} L_{w_{2}}\right)\right) \\
& =\sum_{\left(w_{1}, w_{2}\right) \in \mathbb{G}^{2}} \tau\left(m_{w_{1}}^{(1)} m_{w_{2}}^{(2)} w_{1}^{-1} L_{w_{1}} L_{w_{2}}\right)
\end{aligned}
$$

where

$$
\begin{aligned}
m_{w_{2}}^{(2) w_{1}^{-1}} & =\alpha_{w_{1}}\left(m_{w_{2}}^{(2)}\right) \\
& =\sum_{\left(w_{1}, w_{2}\right) \in \mathbb{G}^{2}, w_{1} w_{2} \neq \emptyset} \operatorname{tr} \circ E\left(m_{w_{1}}^{(1)} m_{w_{2}}^{(2) w_{1}^{-1}} L_{w_{1} w_{2}}\right)
\end{aligned}
$$




$$
\begin{aligned}
& =\sum_{\left(w_{1}, w_{2}\right) \in \mathbb{G}^{2}, w_{1} w_{2} \in V\left(G^{\wedge}\right)} \operatorname{tr}\left(m_{w_{1}}^{(1)} m_{w_{2}}^{(2) w_{1}^{-1}} L_{w_{1} w_{2}}\right) \\
& =\sum_{\left(w_{1}, w_{2}\right) \in \mathbb{G}^{2}, w_{1} w_{2} \in V\left(G^{\wedge}\right)} \operatorname{tr}_{w_{1} w_{2}}\left(m_{w_{1}}^{(1)} m_{w_{2}}^{(2) w_{1}^{-1}} L_{w_{1} w_{2}}\right) \\
& =\sum_{\left(w_{1}, w_{2}\right) \in \mathbb{G}^{2}, w_{1} w_{2} \in V\left(G^{\wedge}\right)} \operatorname{tr}\left(m_{w_{1}}^{(1)} m_{w_{2}}^{(2)} w_{1}^{-1}\right) \\
& =\sum_{\left(w_{1}, w_{2}\right) \in \mathbb{G}^{2}, w_{1} w_{2} \in V\left(G^{\wedge}\right)} \operatorname{tr}\left(m_{w_{1}}^{(1)} \alpha_{w_{1}}\left(m_{w_{2}}^{(2)}\right)\right) .
\end{aligned}
$$

Similarly, we have

$$
\tau\left(a_{2} a_{1}\right)=\sum_{\left(w_{2}, w_{1}\right) \in \mathbb{G}^{2}, w_{2} w_{1} \in V\left(G^{\wedge}\right)} \operatorname{tr}\left(m_{w_{2}}^{(2)} \alpha_{w_{2}}\left(m_{w_{1}}^{(1)}\right)\right) .
$$

Consider the case $w_{1} w_{2} \in V\left(G^{\wedge}\right)$ for $w_{1}, w_{2} \in \mathbb{G}$. By the admissibility and $(\mathrm{RR})$ on $\mathbb{G}$,

$$
w_{1} w_{2} \in V\left(G^{\wedge}\right) \text { if and only if } w_{1}=w_{2}^{-1} \text { in } \mathbb{G} .
$$

For instance, if $w_{1}=v=w_{2}$ in $V\left(G^{\wedge}\right) \subset \mathbb{G}$, then $w_{1} w_{2}=v \Leftrightarrow w_{1}=v=$ $v^{-1}=w_{2}^{-1}$. So, we can re-write (3.2) and (3.3) as follows:

$$
\tau\left(a_{1} a_{2}\right)=\sum_{w \in \mathbb{G} \backslash\{\emptyset\}} \operatorname{tr}\left(m_{w}^{(1)} \alpha_{w^{-1}}\left(m_{w^{-1}}^{(2)}\right)\right)
$$

and

$$
\tau\left(a_{2} a_{1}\right)=\sum_{w \in \mathbb{G} \backslash\{\emptyset\}} \operatorname{tr}\left(m_{w}^{(2)} \alpha_{w^{-1}}\left(m_{w^{-1}}^{(1)}\right)\right) .
$$

Therefore, by $(3.2)^{\prime}$ and $(3.3)^{\prime}$, we obtain that, in general,

$$
\tau\left(a_{1} a_{2}\right) \neq \tau\left(a_{2} a_{1}\right) \text { for } a_{1}, a_{2} \in \mathbb{M}_{G} .
$$

This shows that the linear functional $\tau$ on $\mathbb{M}_{G}$, defined by $\operatorname{Tr} \circ E$, is in general not a trace! And we can realize that:

Observation This non-tracial property of $\tau$ on $\mathbb{M}_{G}$ is completely dependent upon the given groupoid action $\alpha$ of $\mathbb{G}$.

Observe that, for any $a=\sum_{w \in \mathbb{G}} m_{w} L_{w} \in \mathbb{M}_{G}$,

$$
\begin{aligned}
\tau\left(a^{*}\right) & =\tau\left(\sum_{w \in \mathbb{G}}\left(m_{w} L_{w}\right)^{*}\right)=\tau\left(\sum_{w \in \mathbb{G}} L_{w}^{*} m_{w}^{*}\right) \\
& =\tau\left(\sum_{w \in \mathbb{G}} m_{w}^{*} w^{-1} L_{w}^{*}\right)=\sum_{w \in \mathbb{G}} \tau\left(\alpha_{w}\left(m_{w}^{*}\right) L_{w}^{*}\right) \\
& =\sum_{w \in V\left(G^{\wedge}\right)} \tau\left(\alpha_{w}\left(m_{w}^{*}\right) L_{w}^{*}\right)=\sum_{w \in V\left(G^{\wedge}\right)} \operatorname{tr}_{w}\left(\alpha_{w}\left(m_{w}^{*}\right) L_{w}\right)
\end{aligned}
$$




$$
\begin{aligned}
& =\sum_{w \in V\left(G^{\wedge}\right)} \operatorname{tr}\left(\alpha_{w}\left(m_{w}^{*}\right)\right) \\
& =\sum_{w \in V\left(G^{\wedge}\right)} \operatorname{tr}\left(m_{w}^{*}\right) \quad\left(\text { since } \alpha_{v}(m)=m, \forall m \in M \text { and } v \in V\left(G^{\wedge}\right)\right) \\
& =\sum_{w \in V\left(G^{\wedge}\right)} \overline{\operatorname{tr}(m)}=\overline{\tau(a)} .
\end{aligned}
$$

Thus it is easy to check

$$
\tau\left(a^{*}\right)=\overline{\tau(a)} \text { for } a \in \mathbb{M}_{G} .
$$

So, by (3.4) and (3.5), the linear functional $\tau$ on $\mathbb{M}_{G}$ is a continuous linear functional, constructing $\left(\mathbb{M}_{G}, \tau\right)$ be a (non-tracial) scalar-valued $W^{*}$-probability space.

Definition 3.3. Let $\mathbb{M}_{G}=M \times_{\alpha} \mathbb{G}$ be a graph von Neumann algebra. Define the $\alpha$-fixed subalgebra $M^{\alpha}$ of $M$ by

$$
M^{\alpha} \stackrel{\text { def }}{=} v N\left(\left\{m \in M: \alpha_{w}(m)=m, \forall w \in \mathbb{G}\right\}\right) .
$$

Suppose $M=\mathbb{C}$. Then we can easily check that

$$
M^{\alpha}=\mathbb{C}^{\alpha}=\mathbb{C}=M
$$

for all groupoid actions $\alpha$ of $\mathbb{G}$, by the linearity of $\alpha$ on $\mathbb{G}$. Thus, if $M=\mathbb{C}$, we do not need to consider the groupoid-action-fixed property.

Assume now the operators $\alpha_{w}$ 's are identity on $M$ (i.e., $\left.\alpha_{w}\right|_{M}=1_{M}$ for all $w \in \mathbb{G})$, equivalently,

$$
m L_{w} L_{w}^{*}=L_{w}^{*} m L_{w} \text { for all } m \in M .
$$

This represents some kind of symmetry for the cornered $W^{*}$-subalgebras of $\mathbb{M}_{G}$. In this case, we have $M^{\alpha}=M$.

Definition 3.4. Let $\mathbb{M}_{G}=M \times_{\alpha} \mathbb{G}$ be a graph von Neumann algebra. We say that $M$ is $\alpha$-fixed, if the $\alpha$-fixed subalgebra $M^{\alpha}$ of $M$ is identical to $M$, itself, i.e., $M^{\alpha}=M$.

As we observed in the previous paragraph, if $M=\mathbb{C}$, then $M$ is groupoidaction-fixed. Recall that we defined the linear functional $\tau=\operatorname{tr} \circ E$ on a graph von Neumann algebra $\mathbb{M}_{G}=M \times_{\alpha} \mathbb{G}$. Under the additional condition that $M$ is $\alpha$-fixed, this linear functional $\tau$ is a trace on $\mathbb{M}_{G}$.

Proposition 3.1. Let $(M, \operatorname{tr})$ be a tracial $W^{*}$-probability space and let $\mathbb{M}_{G}=$ $M \times_{\alpha} \mathbb{G}$ be a graph von Neumann algebra. Let $\left(\mathbb{M}_{G}, \tau\right)$ be a diagonal graph $W^{*}$-probability space. Assume that $M$ is $\alpha$-fixed $\left(M^{\alpha}=M\right)$. Then the linear functional $\tau=\operatorname{tr} \circ E$ is a well-defined trace on $\mathbb{M}_{G}$. 
Proof. Observe that if $w_{1} w_{2}$ is a vertex in $\mathbb{G}$, then $w_{2} w_{1}$ is a vertex in $\mathbb{G}$, too, where $w_{1}, w_{2} \in \mathbb{G}$. We know that $w_{1} w_{2} \in V\left(G^{\wedge}\right)$ if and only if $w_{1}=w_{2}^{-1}$. So, if we take $w_{1}=w$, then $w_{2}=w^{-1}$. Thus,

$$
w_{1} w_{2}=w w^{-1} \in V\left(G^{\wedge}\right)
$$

and

$$
w_{2} w_{1}=w^{-1} w \in V\left(G^{\wedge}\right) .
$$

Assume now that we have two operators $a_{k}=\sum_{w_{k} \in \mathbb{G}} m_{w_{k}}^{(k)} L_{w_{k}}$ in $\mathbb{M}_{G}$ for $k=1,2$. Then, by $(3.2)^{\prime}$ and $(3.3)^{\prime}$, we can get that

$$
\tau\left(a_{1} a_{2}\right)=\sum_{w \in \mathbb{G} \backslash\{\emptyset\}} \operatorname{tr}\left(m_{w}^{(1)} \alpha_{w^{-1}}\left(m_{w^{-1}}^{(2)}\right)\right),
$$

and

$$
\tau\left(a_{2} a_{1}\right)=\sum_{w^{\prime} \in \mathbb{G} \backslash\{\emptyset\}} \operatorname{tr}\left(m_{w^{\prime}}^{(2)} \alpha_{\left(w^{\prime}\right)^{-1}}\left(m_{\left(w^{\prime}\right)^{-1}}^{(1)}\right)\right) .
$$

Since $M$ is $\alpha$-fixed, by the previous computations, we have

$$
\tau\left(a_{1} a_{2}\right)=\sum_{w \in \mathbb{G} \backslash\{\emptyset\}} \operatorname{tr}\left(m_{w}^{(1)} m_{w^{-1}}^{(2)}\right)
$$

and

$$
\tau\left(a_{2} a_{1}\right)=\sum_{w^{\prime} \in \mathbb{G} \backslash\{\emptyset\}} \operatorname{tr}\left(m_{w^{\prime}}^{(2)} m_{\left(w^{\prime}\right)-1}^{(1)}\right) .
$$

So, $\tau\left(a_{1} a_{2}\right)=\tau\left(a_{2} a_{1}\right)$. Therefore, the continuous linear functional $\tau=\operatorname{tr} \circ E$ is a trace on $\mathbb{M}_{G}$, under the $\alpha$-fixed property of $M$.

For example, if $M=\mathbb{C}$, then $M$ is $\alpha$-fixed for all groupoid actions $\alpha$. Also, if $\alpha_{w}=1_{M}$ for all $w \in \mathbb{G}$, then $M$ is $\alpha$-fixed. So, by the previous proposition, the linear functional $\tau$ on $\mathbb{M}_{G}$ is a trace.

Definition 3.5. If $\tau$ is a trace on $\mathbb{M}_{G}$, then we say that $\left(\mathbb{M}_{G}, \tau\right)$ is a diagonal tracial graph $W^{*}$-probability space.

Let $\mathbb{M}_{G}=M \times_{\alpha} \mathbb{G}$ be a graph von Neumann algebra. Recall that, in [6], we computed the $\mathbb{D}_{G}$-valued $*$-moments

$$
E\left(\left(m_{1} L_{w_{1}}\right) \cdots\left(m_{n} L_{w_{n}}\right)\right)
$$

and the $\mathbb{D}_{G}$-valued $*$-cumulants

$$
k_{n}\left(\left(m_{1} L_{w_{1}}\right), \ldots,\left(m_{n} L_{w_{n}}\right)\right)
$$

of operators $m_{1} L_{w_{1}}, \ldots, m_{n} L_{w_{n}}$ in $\mathbb{M}_{G}$, where $m_{1}, \ldots, m_{n} \in M$ and $w_{1}, \ldots, w_{n}$ $\in \mathbb{G}$ for all $n \in \mathbb{N}$ (Also see Section 2);

$$
E\left(\left(m_{1} L_{w_{1}}\right) \cdots\left(m_{n} L_{w_{n}}\right)\right)=m_{0} E\left(L_{w_{1} \cdots w_{n}}\right)
$$

and

$$
k_{n}\left(\left(m_{1} L_{w_{1}}\right), \ldots,\left(m_{n} L_{w_{n}}\right)\right)=\mu_{0} m_{0} E\left(L_{w_{1} \cdots w_{n}}\right)
$$


where

$$
m_{0}=m_{1} m_{2}^{w_{1}^{-1}} m_{3}^{\left(w_{1} w_{2}\right)^{-1}} \cdots m_{n}^{\left(w_{1} \ldots w_{n-1}\right)^{-1}} \text { in } M,
$$

where $m^{w^{-1}}=\alpha_{w}(m)$ for all $m \in M$ and $w \in \mathbb{G}$, and

$$
\mu_{0}=\sum_{\pi \in N C\left(w_{1}, \ldots, w_{n}\right)} \mu\left(\pi, 1_{n}\right) \text { in } \mathbb{C},
$$

where

$$
N C\left(w_{1}, \ldots, w_{n}\right) \stackrel{\text { def }}{=}\left\{\theta \in N C(n) \mid \begin{array}{c}
E_{\theta}\left(L_{w_{1}}, \ldots, L_{w_{n}}\right) \\
=E\left(L_{w_{1} \cdots w_{n}}\right) \neq 0_{\mathbb{D}_{G}}
\end{array}\right\} .
$$

The above computations make us deal with $\mathbb{D}_{G}$-valued $*$-cumulants easily from $\mathbb{D}_{G}$-valued $*$-moments. Since our trace $\tau$ on $\mathbb{M}_{G}$ is defined by $\operatorname{Tr} \circ E$, we can apply the above $\mathbb{D}_{G}$-valued computations to the scalar-valued case.

Lemma 3.2. Let $m_{1} L_{w_{1}}, \ldots, m_{n} L_{w_{n}}$ be operators in $\mathbb{M}_{G}=M \times_{\alpha} \mathbb{G}$, where $m_{1}, \ldots, m_{n} \in M$ and $w_{1}, \ldots, w_{n} \in \mathbb{G}$. Then

$$
\tau\left(\left(m_{1} L_{w_{1}}\right) \cdots\left(m_{n} L_{w_{n}}\right)\right)= \begin{cases}\operatorname{tr}\left(m_{0}\right) & \text { if } w_{1} \cdots w_{n} \in V\left(G^{\wedge}\right), \\ 0 & \text { otherwise, }\end{cases}
$$

$$
k_{n}^{\tau}\left(\left(m_{1} L_{w_{1}}\right), \ldots,\left(m_{n} L_{w_{n}}\right)\right)=\sum_{\pi \in N C\left(w_{1}, \ldots, w_{n}\right)}\left(\prod_{V \in \pi}\left(\mu_{(V)} \operatorname{tr}\left(m_{(V)}\right)\right)\right) \alpha \mathbb{G}
$$

for all $n \in \mathbb{N}$, where the subset $N C\left(w_{1}, \ldots, w_{n}\right)$ of $N C(n)$ is given in Section 2.3, and where

$$
\begin{aligned}
& m_{0}=m_{1} m_{2}^{w_{1}^{-1}} m_{3}^{\left(w_{1} w_{2}\right)^{-1}} \cdots m_{n}^{\left(w_{1} \ldots w_{n-1}\right)^{-1}} \text { in } M, \\
& \mu_{(V)}=\mu\left(0_{|V|}, 1_{|V|}\right)=\frac{(-1)^{|V|-1}}{|V|}\left(\begin{array}{c}
2(|V|-1) \\
|V|-1
\end{array}\right) \text { in } \mathbb{Z}
\end{aligned}
$$

and

$$
m_{(V)}=m_{j_{1}} m_{j_{2}}^{w_{j_{1}}^{-1}} m_{j_{3}}^{\left(w_{j_{1}} w_{j_{2}}\right)^{-1}} \cdots m_{j_{k}}^{\left(w_{j_{1}} w_{j_{2}} \cdots w_{j_{k-1}}\right)^{-1}} \text { in } M
$$

whenever $V=\left(j_{1}, \ldots, j_{k}\right)$.

Proof. Fix $n \in \mathbb{N}$ and $m_{1} L_{w_{1}}, \ldots, m_{n} L_{w_{n}} \in\left(\mathbb{M}_{G}, \tau\right)$. Then we have that

$$
\begin{aligned}
& \tau\left(m_{1} L_{w_{1}} \cdots m_{n} L_{w_{n}}\right) \\
& =\operatorname{tr}\left(E\left(m_{1} L_{w_{1}} \cdots m_{n} L_{w_{n}}\right)\right) \\
& = \begin{cases}\operatorname{Tr}\left(m_{0} E\left(L_{w_{1} \cdots w_{n}}\right)\right) & \text { if } w_{1} \cdots w_{n} \in V(\hat{G}), \\
\operatorname{Tr}\left(m_{0} \cdot 0_{\mathbb{D}_{G}}\right) & \text { otherwise }\end{cases} \\
& = \begin{cases}\operatorname{Tr}_{\left|V\left(G^{\wedge}\right)\right|}\left(\left(\underset{v \in V\left(G^{\wedge}\right)}{\oplus} \operatorname{tr}_{v}\right)\left(m_{0} L_{w_{1} \cdots w_{n}}\right)\right) & \text { if } w_{1} \cdots w_{n} \in V\left(G^{\wedge}\right), \\
\operatorname{Tr}\left(0_{\mathbb{D}_{G}}\right)=0 & \text { otherwise }\end{cases}
\end{aligned}
$$




$$
\begin{aligned}
& =\operatorname{tr}_{\left|V\left(G^{\wedge}\right)\right|}\left(\operatorname{tr}_{w_{1} \cdots w_{n}}\left(m_{0} L_{w_{1} \cdots w_{n}}\right)\right) \\
& =\operatorname{tr}_{\left|V\left(G^{\wedge}\right)\right|}\left(\operatorname{tr}\left(m_{0}\right)\right)=\operatorname{tr}\left(m_{0}\right),
\end{aligned}
$$

where $m_{0}=m_{1} m_{2}^{w_{1}^{-1}} m_{3}^{\left(w_{1} w_{2}\right)^{-1}} \cdots m_{n}^{\left(w_{1} \cdots w_{n-1}\right)^{-1}}$ in $M$.

Now, compute

$$
\begin{aligned}
& k_{n}^{\tau}\left(\left(m_{1} L_{w_{1}}\right), \ldots,\left(m_{n} L_{w_{n}}\right)\right) \\
= & \sum_{\pi \in N C(n)} \tau_{\pi}\left(m_{1} L_{w_{1}} \cdots m_{n} L_{w_{n}}\right) \mu\left(\pi, 1_{n}\right) \\
= & \sum_{\pi \in N C(n)}\left(\prod_{V \in \pi} \tau_{V}\left(m_{1} L_{w_{1}}, \ldots, m_{n} L_{w_{n}}\right)\right) \mu\left(\pi, 1_{n}\right) \\
= & \sum_{\pi \in N C(n)}\left(\prod_{V \in \pi} \tau_{V}\left(m_{1} L_{w_{1}}, \ldots, m_{n} L_{w_{n}}\right) \mu\left(0_{|V|}, 1_{|V|}\right)\right)
\end{aligned}
$$

since there is no insertion property on the computations of $*$-cumulants on $\left(\mathbb{M}_{G}, \tau\right)$ and since the Möbius functional $\mu$ is multiplicative (See [16]), where $|V|$ means the size of the block $V$,

$$
\begin{aligned}
& = \begin{cases}\sum_{\pi \in N C(n)}\left(\prod_{V \in \pi}\left(\mu_{(V)} \operatorname{tr}\left(m_{(V)}\right)\right)\right) & \text { if } w_{(V)} \in V\left(G^{\wedge}\right) \\
0 & \text { otherwise, }\end{cases} \\
& =\sum_{\pi \in N C\left(w_{1}, \ldots, w_{n}\right)}\left(\prod_{V \in \pi}\left(\mu_{(V)} \operatorname{tr}\left(m_{(V)}\right)\right)\right) \alpha \mathbb{G}
\end{aligned}
$$

by (3.9), where

$$
\begin{gathered}
w_{(V)}=w_{j_{1}} w_{j_{2}} \cdots w_{j_{k}} \text { in } \mathbb{G} \\
\mu_{(V)}=\mu\left(0_{|V|}, 1_{|V|}\right)=\frac{(-1)^{|V|-1}}{|V|}\left(\begin{array}{c}
2(|V|-1) \\
|V|-1
\end{array}\right) \text { in } \mathbb{C},
\end{gathered}
$$

since $\mu\left(0_{k}, 1_{k}\right)=(-1)^{k-1} c_{k-1}$ for all $k \in \mathbb{N}$, where $c_{m} \stackrel{\text { def }}{=} \frac{1}{m+1}\left(\begin{array}{c}2 m \\ m\end{array}\right)$ is the $m$-th Catalan number for all $m \in \mathbb{N}$,

$$
m_{(V)}=m_{j_{1}} m_{j_{2}}^{w_{j_{2}}^{-1}} m_{j_{3}}^{\left(w_{j_{2}} w_{j_{3}}\right)^{-1}} \cdots m_{j_{k}}^{\left(w_{j_{1}} \cdots w_{j_{k-1}}\right)^{-1}} \text { in } M,
$$

whenever $V=\left(j_{1}, \ldots, j_{k}\right) \in \pi$ are blocks.

Corollary 3.3. Assume that a given von Neumann algebra $M$ is $\alpha$-fixed, and hence $\left(\mathbb{M}_{G}, \tau\right)$ is a diagonal "tracial" graph $W^{*}$-probability space. Let $m_{1}$ $L_{w_{1}}, \ldots, m_{n} L_{w_{n}}$ be free random variables in $\left(\mathbb{M}_{G}, \tau\right)$ for $n \in \mathbb{N}$. Then we have that:

$$
\tau\left(\left(m_{1} L_{w_{1}}\right) \cdots\left(m_{n} L_{w_{n}}\right)\right)= \begin{cases}\operatorname{tr}\left(m_{1} m_{2} \cdots m_{n}\right) & \text { if } w_{1} \ldots w_{n} \in V\left(G^{\wedge}\right), \\ 0 & \text { otherwise. }\end{cases}
$$


$(3.8)^{\prime}$

$$
k_{n}^{\tau}\left(\left(m_{1} L_{w_{1}}\right), \ldots,\left(m_{n} L_{w_{n}}\right)\right)=\sum_{\pi \in N C\left(w_{1}, \ldots, w_{n}\right)}\left(\prod_{V \in \pi}\left(\operatorname{tr}\left(m_{(V)}\right)\right)\right) \mu\left(\pi, 1_{n}\right),
$$

where $N C\left(w_{1}, \ldots, w_{n}\right), w_{(V)} \in \mathbb{G}$ and $\mu_{(V)} \in \mathbb{Z}$ are given as in the previous lemma.

Proof. The computation (3.7)' is trivial, by (3.7). So, it suffices to show the computation $(3.8)^{\prime}$ holds. Compute

$$
\begin{aligned}
& k_{n}^{\tau}\left(\left(m_{1} L_{w_{1}}\right), \ldots,\left(m_{n} L_{w_{n}}\right)\right) \\
= & \sum_{\pi \in N C\left(w_{1}, \ldots, w_{n}\right)}\left(\prod_{V \in \pi}\left(\mu_{(V)} \operatorname{tr}\left(m_{(V)}\right)\right)\right)
\end{aligned}
$$

(by $(3.8)$, where $\mu_{(V)}$ and $m_{(V)}$ are given as in the previous lemma)

$$
=\sum_{\pi \in N C\left(w_{1}, \ldots, w_{n}\right)}\left(\prod_{V \in \pi}\left(\operatorname{tr}\left(m_{(V)}\right)\right)\right) \mu\left(\pi, 1_{n}\right)
$$

The formula $(3.8)^{\prime}$ shows the relation between

$$
k_{n}^{\tau}\left(\left(m_{1} L_{w_{1}}\right), \ldots,\left(m_{n} L_{w_{n}}\right)\right) \text { and } k_{n}^{\text {tr }}\left(m_{1}, \ldots, m_{n}\right),
$$

under the admissibility of $\mathbb{G}$. Recall that

$$
k_{n}^{\operatorname{tr}}\left(m_{1}, \ldots, m_{n}\right)=\sum_{\theta \in N C(n)}\left(\prod_{B \in \theta} \operatorname{tr}\left(m_{(B)}\right)\right) \mu\left(\theta, 1_{n}\right) .
$$

And, by $(3.8)^{\prime}$,

$$
k_{n}^{\tau}\left(\left(m_{1} L_{w_{1}}\right), \ldots,\left(m_{n} L_{w_{n}}\right)\right)=\sum_{\pi \in N C\left(w_{1}, \ldots, w_{n}\right)}\left(\prod_{V \in \pi} \operatorname{tr}\left(m_{(V)}\right)\right) \mu\left(\pi, 1_{n}\right) .
$$

Thus, the $\mathbb{C}$-valued freeness on $\left(\mathbb{M}_{G}, \tau\right)$ is determined by the $\mathbb{C}$-valued freeness on $(M, \operatorname{tr})$ "and" the admissibility on $\mathbb{G}$.

The following proposition is the direct consequence of (3.7).

Proposition 3.4. The $M$-diagonal graph $W^{*}$-probability space $\left(\mathbb{M}_{G}, E\right)$ and the diagonal graph $W^{*}$-probability space $\left(\mathbb{M}_{G}, \tau\right)$ are compatible.

Proof. Let $x=\sum_{w \in \mathbb{G}} m_{w} L_{w}$ be an operator in $\mathbb{M}_{G}$. Then

$$
\tau(x)=\sum_{v \in V\left(G^{\wedge}\right)} \operatorname{tr}\left(m_{v}\right) .
$$

Also, we can have that $E(x)=\sum_{v \in V\left(G^{\wedge}\right)} m_{v} L_{v}$. So,

$$
\tau(E(x))=\tau\left(\sum_{v \in V\left(G^{\wedge}\right)} m_{v} L_{v}\right)=\sum_{v \in V\left(G^{*}\right)} \tau\left(m_{v} L_{v}\right)
$$




$$
\begin{aligned}
& =\sum_{v \in V\left(G^{\wedge}\right)} \operatorname{Tr}\left(E\left(m_{v} L_{v}\right)\right)=\sum_{v \in V\left(G^{\wedge}\right)} \operatorname{tr}\left(m_{v} L_{v}\right) \\
& =\sum_{v \in V\left(G^{\wedge}\right)} \operatorname{Tr}_{\left|V\left(G^{\wedge}\right)\right|}\left(\left(\underset{v^{\prime} \in V\left(G^{\wedge}\right)}{\oplus} \operatorname{tr}_{v^{\prime}}\right)\left(m_{v} L_{v}\right)\right) \\
& =\sum_{v \in V\left(G^{\wedge}\right)} \operatorname{Tr}_{\left|V\left(G^{\wedge}\right)\right|}\left(\operatorname{tr}_{v}\left(m_{v} L_{v}\right)\right)=\sum_{v \in V\left(G^{\wedge}\right)} \operatorname{tr}_{\left|V\left(G^{\wedge}\right)\right|}\left(\operatorname{tr}\left(m_{v}\right)\right) \\
& =\sum_{v \in V\left(G^{*}\right)} \operatorname{tr}\left(m_{v}\right) .
\end{aligned}
$$

Remark 3.1. The above compatibility of $\left(\mathbb{M}_{G}, E\right)$ and $\left(\mathbb{M}_{G}, \tau\right)$ explains that the $\mathbb{D}_{G}$-valued free distributional data determined by the canonical conditional expectation $E$ and the scalar-valued free distributional data determined by the linear functional $\tau$ of the operators in $\mathbb{M}_{G}$ are closely related.

Let $\left(\mathbb{M}_{G}, E\right)$ be a $M$-diagonal graph $W^{*}$-probability space and let $x_{1}, \ldots, x_{s}$ $\in\left(\mathbb{M}_{G}, E\right)$ be $\mathbb{D}_{G^{-}}$valued random variables. Then there always exists a $\mathbb{D}_{G^{-}}$ valued free distribution of $x_{1}, \ldots, x_{s}$ (See the more general cases in [32], [31], and $[16])$;

defined by

$$
\sigma_{x_{1}, \ldots, x_{s}}: \mathbb{D}_{G}\left[z_{1}, \ldots, z_{2 s}\right] \rightarrow \mathbb{D}_{G}
$$

$$
\sigma_{x_{1}, \ldots, x_{s}}\left(P\left(z_{1}, \ldots, z_{2 s}\right)\right)=E\left(P\left(x_{1}, x_{1}^{*}, \ldots, x_{s}, x_{s}^{*}\right)\right)
$$

for all $P \in \mathbb{D}_{G}\left[z_{1}, \ldots, z_{2 s}\right]$, where $\mathbb{D}_{G}\left[z_{1}, \ldots, z_{2 s}\right]$ is the collection of all functions having their forms

$$
P\left(z_{1}, \ldots, z_{2 s}\right)=d_{0}+\sum_{n=1}^{N} \sum_{\left(j_{1}, \ldots, j_{n}\right) \in\{1, \ldots, 2 s\}^{n}}\left(d_{j_{1}, \ldots, j_{n}}\right) z_{j_{1}} \cdots z_{j_{n}}
$$

with

$$
d_{0}, d_{j_{1}, \ldots, j_{n}} \in \mathbb{D}_{G} \text { for } N \in \mathbb{N}
$$

and where $z_{1}, \ldots, z_{2 s}$ are arbitrary noncommutative indeterminents (i.e., $\mathbb{D}_{G}\left[z_{1}\right.$, $\left.\ldots, z_{2 s}\right]$ is a $\mathbb{D}_{G}-\mathbb{D}_{G}$-bimodule $\mathbb{D}_{G} X_{\mathbb{D}_{G}}$, with $\left.X=\left\{z_{1}, \ldots, z_{2 s}\right\}\right)$.

The $\mathbb{D}_{G}$-valued free distribution $\sigma_{x_{1}, \ldots, x_{s}}$ of the operators $x_{1}, \ldots, x_{s} \in \mathbb{M}_{G}$ is characterized by the joint $*$-moments of them or equivalently the joint $*$ cumulants of them, by the Möbius inversion. In particular, if we have

$$
P\left(z_{1}, \ldots, z_{2 s}\right)=z_{i_{1}} \cdots z_{i_{n}} \text { in } \mathbb{D}_{G}\left[z_{1}, \ldots, z_{2 s}\right],
$$

we can get the $\left(i_{1}, \ldots, i_{n}\right)$-th joint $*$-moment

$$
\sigma_{x_{1}, \ldots, x_{s}}(P)=E\left(x_{i_{1}}^{r_{1}} \cdots x_{i_{n}}^{r_{n}}\right)
$$

with

$$
r_{k}= \begin{cases}1 & \text { if } i_{k} \text { is odd } \\ * & \text { if } i_{k} \text { is even }\end{cases}
$$


for all $\left(i_{1}, \ldots, i_{n}\right) \in\{1, \ldots, 2 s\}^{n}$ for $n \in \mathbb{N}$. Since $E$ has the bimodule map property, to compute $E\left(x_{i_{1}}^{r_{1}} \cdots x_{i_{n}}^{r_{n}}\right)$, we need to compute $E\left(m_{1} L_{w_{1}} \cdots m_{n} L_{w_{n}}\right)$, where $m_{1}, \ldots, m_{n} \in M$ and $w_{1}, \ldots, w_{n} \in \mathbb{G}$. And we already know

$$
E\left(m_{1} L_{w_{1}} \cdots m_{n} L_{w_{n}}\right)=m_{0} E\left(L_{w_{1} \cdots w_{n}}\right),
$$

where $m_{0}=m_{1} m_{2}^{w_{1}^{-1}} m_{3}^{\left(w_{1} w_{2}\right)^{-1}} \cdots m_{n}^{\left(w_{1} \cdots w_{n-1}\right)^{-1}}$ in $M$.

Similarly, the above operators $x_{1}, \ldots, x_{s}$ of $\mathbb{M}_{G}$ have (scalar-valued) free distribution of them, by regarding them as random variables in the diagonal graph $W^{*}$-probability space $\left(\mathbb{M}_{G}, \tau\right)$,

$$
\nu_{x_{1}, \ldots, x_{s}}: \mathbb{C}\left[t_{1}, \ldots, t_{2 s}\right] \rightarrow \mathbb{C},
$$

defined by a linear functional

$$
\nu_{x_{1}, \ldots, x_{s}}\left(Q\left(t_{1}, \ldots, t_{2 s}\right)\right)=\tau\left(Q\left(x_{1}, x_{1}^{*}, \ldots, x_{s}, x_{s}^{*}\right)\right)
$$

for all $Q \in \mathbb{C}\left[t_{1}, \ldots, t_{2 s}\right]$, where $\mathbb{C}\left[t_{1}, \ldots, t_{2 s}\right]$ is the collection of all functions having their forms

$$
Q\left(t_{1}, \ldots, t_{2 s}\right)=r_{0}+\sum_{n=1}^{N} \sum_{\left(i_{1}, \ldots, i_{n}\right) \in\{1, \ldots, s\}^{n}}\left(r_{i_{1}, \ldots, i_{n}}\right) t_{i_{1}} \ldots t_{i_{n}}
$$

for $N \in \mathbb{N}$, where $r_{0}, r_{i_{1}, \ldots, i_{n}} \in \mathbb{C}$ and $t_{1}, \ldots, t_{2 s}$ are the noncommutative indeterminents.

By the compatibility of $\left(\mathbb{M}_{G}, E\right)$ and $\left(\mathbb{M}_{G}, \tau\right)$, and by the admissibility on $\mathbb{G}$, we can get the following theorem.

Theorem 3.5. Let $G$ be a finite directed graph with its graph groupoid $\mathbb{G}$ and $\mathbb{M}_{G}=M \times_{\alpha} \mathbb{G}$, a graph von Neumann algebra and let $\left(\mathbb{M}_{G}, E\right)$ and $\left(\mathbb{M}_{G}, \tau\right)$ be the $M$-diagonal graph $W^{*}$-probability space and the diagonal graph $W^{*}$-probability space, respectively. Let $x_{1}, \ldots, x_{s} \in \mathbb{M}_{G}$ be operators and let $\sigma_{x_{1}, \ldots, x_{s}}$ and $\nu_{x_{1}, \ldots, x_{s}}$, be the $\mathbb{D}_{G}$-valued free distribution and the scalar-valued free distribution of $x_{1}, \ldots, x_{s}$, respectively, where $s \in \mathbb{N}$. If

$$
P\left(z_{1}, \ldots, z_{2 s}\right)=d_{0}+\sum_{n=1}^{N} \sum_{\left(i_{1}, \ldots, i_{n}\right) \in\{1, \ldots, s\}^{n}}\left(d_{i_{1}, \ldots, i_{n}}\right) z_{i_{1}} \cdots z_{i_{n}}
$$

in $\mathbb{D}_{G}\left[z_{1}, \ldots, z_{2 s}\right]$, then there exists a unique element $Q$ in $\mathbb{C}\left[t_{1}, \ldots, t_{2 s}\right]$ such that

$$
\nu_{x_{1}, \ldots, x_{s}}\left(Q\left(t_{1}, \ldots, t_{2 s}\right)\right)=\operatorname{tr}\left(\sigma_{x_{1}, \ldots, x_{s}}\left(P\left(z_{1}, \ldots, z_{2 s}\right)\right)\right) .
$$

Proof. It is sufficient to consider the case where $x_{j}=m_{j} L_{w_{j}}$ for all $j=1, \ldots, s$, because every operator $x$ in $\mathbb{M}_{G}$ has its expression $\sum_{w \in \mathbb{G}} m_{w} L_{w}$. Put

$$
x_{j}=m_{j} L_{w_{j}} \text { for all } j=1, \ldots, s \text {. }
$$


As in the previous paragraphs, there are the corresponding $\mathbb{D}_{G}$-valued free distribution $\sigma_{x_{1}, \ldots, x_{s}}$ and the free distribution $\nu_{x_{1}, \ldots, x_{s}}$ of the operators $x_{1}, \ldots$, $x_{s}$. Now, let $P \in \mathbb{D}_{G}\left[z_{1}, \ldots, z_{2 s}\right]$ be defined by

$$
P\left(z_{1}, \ldots, z_{2 s}\right)=\left(d_{i_{1}, \ldots, i_{n}}\right) z_{i_{1}} \cdots z_{i_{n}} \text { for } d_{i_{1}, \ldots, i_{n}} \in \mathbb{D}_{G} .
$$

Then we have

$$
\begin{aligned}
\sigma_{x_{1}, \ldots, x_{s}}\left(P\left(z_{1}, \ldots, z_{2 s}\right)\right) & =E\left(P\left(x_{1}, x_{1}^{*}, x_{2}, x_{2}^{*}, \ldots, x_{s}, x_{s}^{*}\right)\right) \\
& =E\left(\left(d_{i_{1}, \ldots, i_{s}}\right)\left(a_{i_{1}} L_{y_{i_{1}}} \cdots a_{i_{n}} L_{y_{i_{n}}}\right)\right)
\end{aligned}
$$

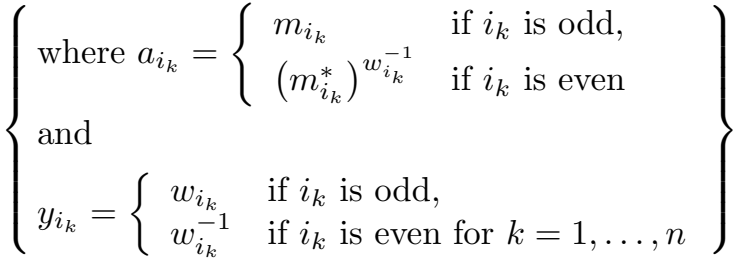

$$
\begin{aligned}
& =E\left(\left(d_{i_{1}, \ldots, i_{n}}\right)\left(a_{0} L_{y_{i_{1}} \cdots y_{i_{n}}}\right)\right) \text {, }
\end{aligned}
$$

where

$$
a_{0}=a_{i_{1}} a_{i_{2}}^{w_{i_{1}}^{-1}} a_{i_{3}}^{\left(w_{i_{1}} w_{i_{2}}\right)^{-1}} \cdots a_{i_{n}}^{\left(w_{i_{1}} \cdots w_{i_{n-1}}\right)^{-1}} \text { in } M .
$$

Clearly, if $y_{i_{1}}, \ldots, y_{i_{n}}$ are not admissible, then (3.10) vanishes. Suppose now that they are admissible, i.e., assume that $y_{i_{1}} \cdots y_{i_{n}} \neq \emptyset$ in $\mathbb{G}$. Denote this nonempty element $y_{i_{1}} \cdots y_{i_{n}}$ by $y_{0}$. Now, let

$$
d_{i_{1}, \ldots, i_{n}}=\sum_{v \in V\left(G^{\wedge}\right)} m_{v} L_{v} \text { in } \mathbb{D}_{G}
$$

and assume that $y_{0}=v_{0} y_{0} v_{0}^{\prime}$ with $v_{0}, v_{0}^{\prime} \in V\left(G^{\wedge}\right)$. Then (3.10) is equal to:

$$
\begin{aligned}
& =E\left(\left(d_{i_{1}, \ldots, i_{n}}\right) a_{0} L_{y_{0}}\right)=E\left(\left(\sum_{v \in V\left(G^{\wedge}\right)} m_{v} L_{v}\right) a_{0} L_{y_{0}}\right) \\
& =\sum_{v \in V\left(G^{\wedge}\right)} E\left(\left(m_{v} L_{v}\right)\left(a_{0} L_{y_{0}}\right)\right)=\sum_{v \in V\left(G^{\wedge}\right)} E\left(m_{v} a_{0} L_{v} L_{y_{0}}\right) \\
& =E\left(m_{v_{0}} a_{0} L_{v_{0}} L_{y_{0}}\right) \\
& = \begin{cases}E\left(\left(m_{v_{0}} a_{0}\right) L_{y_{0}}\right) & \text { if } m_{v_{0}} \neq 0_{M}, \\
0_{\mathbb{D}_{G}} & \text { otherwise. }\end{cases}
\end{aligned}
$$

By (3.11), we can get that

$$
\sigma_{x_{1}, \ldots, x_{s}}\left(d_{i_{1}, \ldots, i_{n}} z_{i_{1}} \cdots z_{i_{n}}\right)= \begin{cases}m_{v_{0}} a_{0} E\left(L_{y_{0}}\right) & \text { if } m_{v_{0}} \neq 0, \\ 0_{\mathbb{D}_{G}} & \text { if } m_{v_{0}}=0 .\end{cases}
$$

Therefore, there exists an element

$$
Q\left(t_{1}, \ldots, t_{2 s}\right)=\left(r_{v_{0}}\right) t_{i_{1}} \cdots t_{i_{n}} \in \mathbb{C}\left[t_{1}, \ldots, t_{2 s}\right],
$$


with $r_{v_{0}} \in \mathbb{C}$, such that

$$
\begin{aligned}
\nu_{x_{1}, \ldots, x_{s}}\left(Q\left(t_{1}, \ldots, t_{2 s}\right)\right) & =\tau\left(Q\left(x_{1}, x_{1}^{*}, \ldots, x_{s}, x_{s}^{*}\right)\right) \\
& =\tau\left(r_{v_{0}}\left(a_{i_{1}} L_{y_{i_{1}}}\right) \cdots\left(a_{i_{n}} L_{y_{i_{n}}}\right)\right) \\
& =r_{v_{0}} \tau\left(a_{0} L_{y_{0}}\right)
\end{aligned}
$$

by using the same notations as above. Therefore, we can have that (3.13) is identified with

$$
r_{v_{0}} \operatorname{tr}\left(E\left(a_{0} L_{y_{0}}\right)\right)=\operatorname{tr}\left(r_{v_{0}}\left(E\left(a_{0} L_{y_{0}}\right)\right)\right) .
$$

Now, we can assume $r_{v_{0}}=\operatorname{tr}\left(m_{v_{0}}\right)$. By the faithfulness of tr, the elements $r_{v_{0}}$ and $m_{v_{0}}$ are uniquely determined from each other, via tr. This shows that

$$
\nu_{x_{1}, \ldots, x_{s}}\left(Q\left(t_{1}, \ldots, t_{2 s}\right)\right)=\operatorname{tr}\left(\sigma_{x_{1}, \ldots, x_{s}}\left(P\left(z_{1}, \ldots, z_{2 s}\right)\right)\right) .
$$

\section{Groupoid $W^{*}$-algebras $\overline{\mathbb{C}[\mathbb{G}]}$ w}

In this section, we will consider graph von Neumann algebras $\mathbb{M}_{G}=M \times_{\alpha}$ $\mathbb{G}$, where the fixed von Neumann algebra $M$ is identical to $\mathbb{C}$. As we observed in Section 2.3, every graph von Neumann algebra $\mathbb{M}_{G}=\mathbb{C} \times_{\alpha} \mathbb{G}$ is $*$-isomorphic to the groupoid $W^{*}$-algebra $\overline{\mathbb{C}[\mathbb{G}]}$ in $B\left(H_{G}\right)$, generated by the graph groupoid $\mathbb{G}$ for all groupoid actions $\alpha$, where $H_{G}$ is the graph Hilbert space induced by $G$.

Notation. To distinguish the difference between the general case $\mathbb{M}_{G}=M \times_{\alpha}$ $\mathbb{G}$, we denote $\overline{\mathbb{C}[\mathbb{G}]}$ by $M_{G}$. We also denote the $\mathbb{C}$-diagonal subalgebra $\mathbb{D}_{G}=$ $\oplus \quad\left(\mathbb{C} \cdot L_{v}\right)$ of $M_{G}$ by $D_{G}$. This groupoid $W^{*}$-algebra $M_{G}$ is called "the" $v \in V\left(G^{\wedge}\right)$ graph von Neumann algebra of $G$.

Remark that, in the case where $M=\mathbb{C}$, the trace $\operatorname{Tr}$ on the $\mathbb{C}$-diagonal subalgebra $D_{G}$ of $M_{G}$ is nothing but the usual trace $\operatorname{Tr}_{\left|V\left(G^{\wedge}\right)\right|}$ on the matricial algebra $M_{\left|V\left(G^{\wedge}\right)\right|}(\mathbb{C})$, and $D_{G}$ is $*$-isomorphic to the subalgebra of $M_{\left|V\left(G^{\wedge}\right)\right|}(\mathbb{C})$ generated by all diagonal matrices (Recall that all graphs in this paper are automatically assumed to be finite). Therefore, we can get the following result:

Theorem 4.1. Let $G$ be a finite directed graph with its graph groupoid $\mathbb{G}$ and let $M_{G}$ be the graph von Neumann algebra $\overline{\mathbb{C}[\mathbb{G}]}$ of $G$ in $B\left(H_{G}\right)$. Then the diagonal graph $W^{*}$-probability space $\left(M_{G}, \tau\right)$ is always tracial.

Proof. It is sufficient to show that the linear functional $\tau: M_{G} \rightarrow \mathbb{C}$ is a trace. Suppose $x_{k}=\sum_{w_{k} \in \mathbb{G}} t_{w_{k}}^{(k)} L_{w_{k}}$ are operators in $M_{G}$ for $k=1,2$. Then

$$
\tau\left(x_{1} x_{2}\right)=\tau\left(\left(\sum_{w_{1} \in \mathbb{G}} t_{w_{1}}^{(1)} L_{w_{1}}\right)\left(\sum_{w_{2} \in \mathbb{G}} t_{w_{2}}^{(2)} L_{w_{2}}\right)\right)
$$




$$
\begin{aligned}
& =\tau\left(\sum_{\left(w_{1}, w_{2}\right) \in \mathbb{G}^{2}}\left(t_{w_{1}}^{(1)} L_{w_{1}}\right)\left(t_{w_{2}}^{(2)} L_{w_{2}}\right)\right) \\
& =\sum_{\left(w_{1}, w_{2}\right) \in \mathbb{G}^{2}} \tau\left(\left(t_{w_{1}}^{(1)} L_{w_{1}}\right)\left(t_{w_{2}}^{(2)} L_{w_{2}}\right)\right) \\
& =\sum_{\left(w_{1}, w_{2}\right) \in \mathbb{G}^{2}} \tau\left(t_{w_{1}}^{(1)} t_{w_{2}}^{(2)} L_{w_{1} w_{2}}\right)
\end{aligned}
$$

(since $t L_{w}=L_{w} t$ for all $t \in \mathbb{C}$ and $w \in \mathbb{G}$ )

$$
=\sum_{\left(w_{1}, w_{2}\right) \in \mathbb{G}^{2}} \tau\left(t_{w_{1}}^{(1)} t_{w_{2}}^{(2)} E\left(L_{w_{1} w_{2}}\right)\right)
$$

(by the compatibility of $\left(M_{G}, E\right)$ and $\left(M_{G}, \tau\right)$, where $E: M_{G} \rightarrow D_{G}$ is the canonical conditional expectation)

$$
\begin{aligned}
& =\sum_{\left(w_{1}, w_{2}\right) \in \mathbb{G}^{2}, w_{1} w_{2} \in V\left(G^{\circ}\right)} \tau\left(t_{w_{1}}^{(1)} t_{w_{2}}^{(2)} E\left(L_{w_{1} w_{2}}\right)\right) \\
& =\sum_{\left(w_{1}, w_{2}\right) \in \mathbb{G}^{2}, w_{1} w_{2} \in V\left(G^{\prime}\right)} \operatorname{tr}\left(t_{w_{1}}^{(1)} t_{w_{2}}^{(2)} E\left(L_{w_{1} w_{2}}\right)\right)
\end{aligned}
$$

(by the definition of $\tau=\operatorname{tr} \circ E$ )

$$
=\sum_{\left(w_{1}, w_{2}\right) \in \mathbb{G}^{2}, w_{1} w_{2} \in V\left(G^{\circ}\right)} \operatorname{tr}\left(t_{w_{1}}^{(1)} t_{w_{2}}^{(2)} L_{w_{1} w_{2}}\right)
$$

(since $E\left(L_{w_{1} w_{2}}\right)=L_{w_{1} w_{2}}$, whenever $w_{1} w_{2} \in V\left(G^{\wedge}\right)$

$$
\begin{aligned}
& =\sum_{\left(w_{1}, w_{2}\right) \in \mathbb{G}^{2}, w_{1} w_{2} \in V\left(G^{\wedge}\right)} t_{w_{1}}^{(1)} t_{w_{2}}^{(2)} \\
& =\sum_{w \in \mathbb{G} \backslash\{\emptyset\}} t_{w}^{(1)} t_{w^{-1}}^{(2)}=\sum_{w^{\prime} \in \mathbb{G} \backslash\{\emptyset\}} t_{w^{\prime}}^{(2)} t_{\left(w^{\prime}\right)^{-1}}^{(1)} \\
& =\sum_{\left(w_{1}, w_{2}\right) \in \mathbb{G}^{2}, w_{1} w_{2} \in V\left(G^{\wedge}\right)} t_{w_{2}}^{(2)} t_{w_{1}}^{(1)} \\
& =\sum_{\left(w_{2}, w_{1}\right) \in \mathbb{G}^{2}, w_{2} w_{1} \in V\left(G^{\wedge}\right)} t_{w_{2}}^{(2)} t_{w_{1}}^{(1)} .
\end{aligned}
$$

The equality in the formulae (4.1) holds, since the subsets

$$
\mathcal{I}_{1}=\left\{\left(w_{1}, w_{2}\right) \in \mathbb{G}^{2}: w_{1} w_{2} \in V\left(G^{\wedge}\right)\right\}
$$

and

$$
\mathcal{I}_{2}=\left\{\left(w_{2}, w_{1}\right) \in \mathbb{G}^{2}: w_{2} w_{1} \in V\left(G^{\wedge}\right)\right\}
$$

in $\mathbb{G}^{2}$ are bijective (See Section: $w_{1} w_{2} \in V\left(G^{\wedge}\right)$, if and only if $w_{1}=w_{2}^{-1}$, if and only if $w_{2} w_{1} \in V\left(G^{\wedge}\right)$. Of course, the vertices $w_{1} w_{2}$ and $w_{2} w_{1}$ are not 
necessarily identical, but it makes $\tau\left(L_{w_{1} w_{2}}\right)=1=\tau\left(L_{w_{2} w_{1}}\right)$, because

$$
\tau\left(L_{v}\right)=\operatorname{tr}\left(E\left(L_{v}\right)\right)=\operatorname{tr}\left(L_{v}\right)=\operatorname{tr}_{v}\left(L_{v}\right)=1 \cdot L_{v}=\operatorname{tr}(1) L_{v}
$$

for all $\left.v \in V\left(G^{\wedge}\right)\right)$. Therefore,

$$
\begin{aligned}
& =\sum_{\left(w_{2}, w_{1}\right) \in \mathbb{G}^{2}, w_{2} w_{1} \in V\left(G^{\wedge}\right)} \tau\left(t_{w_{2}}^{(2)} t_{w_{1}}^{(1)} E\left(L_{w_{2} w_{1}}\right)\right) \\
& =\sum_{\left(w_{2}, w_{1}\right) \in \mathbb{G}^{2}, w_{2} w_{1} \in V\left(G^{\wedge}\right)} \tau\left(t_{w_{2}}^{(2)} t_{w_{1}}^{(1)} L_{w_{2} w_{1}}\right) \\
& =\tau\left(\sum_{\left(w_{2}, w_{1}\right) \in \mathbb{G}^{2}}\left(t_{w_{2}}^{(2)} L_{w_{2}}\right)\left(t_{w_{1}}^{(1)} L_{w_{1}}\right)\right) \\
& =\tau\left(x_{2} x_{1}\right) .
\end{aligned}
$$

The above theorem shows that if $\left(M_{G}, \tau\right)$ is the diagonal graph $W^{*}$-probability space, where $M_{G}$ is the graph von Neumann algebra of $G$, then $\tau$ is always tracial, even if $E: M_{G} \rightarrow D_{G}$ is non-tracial.

\section{An example}

Let $F_{N}$ be the free group with $N$-generators, and let $L\left(F_{N}\right)$ be the group von Neumann algebra $v N\left(F_{N}\right)={\overline{\mathbb{C}}\left[F_{N}\right]}^{w}$ in $B\left(l^{2}\left(F_{N}\right)\right)$, generated by $F_{N}$, where $l^{2}\left(F_{N}\right)$ is the group Hilbert space induced by $F_{N}$. It is well-known that a group von Neumann algebra $L(\Gamma)$ is a factor, if and only if the group $\Gamma$ is an i.c.c group (or an infinite conjugacy class group). Since $F_{N}$ is an i.c.c group, the group von Neumann algebra $L\left(F_{N}\right)$ is a factor. So, we call $L\left(F_{N}\right)$, a free group factor. Recall that a von Neumann algebra $A \subseteq B(K)$ is a factor, if $A^{\prime} \cap A \stackrel{\text { *-isomorphic }}{=} \mathbb{C} \cdot 1_{A}$, where

$$
A^{\prime} \stackrel{\text { def }}{=}\{a \in B(K): a b=b a, \forall a \in A\} .
$$

Free group factors have been studied by various operator algebraists, in particular, free probabilists. We still don't know $L\left(F_{2}\right)$ and $L\left(F_{3}\right)$ are *-isomorphic or not. The famous Radulescu's theorem says that: either (i) or (ii) holds true, where

$$
\begin{aligned}
& \text { (i) } L\left(F_{n}\right) \stackrel{\text { *-isomorphic }}{=} L\left(F_{\infty}\right) \text { for all } n \in \mathbb{N} \backslash\{1\}, \\
& \text { (ii) } L\left(F_{k_{1}}\right) \stackrel{\text { *-isomorphic }}{\neq} L\left(F_{k_{2}}\right) \text {, whenever } k_{1} \neq k_{2} \text { in } \mathbb{N} \cup\{\infty\} \text { (See [18]). }
\end{aligned}
$$

So, the study of free group factors is very interesting and important in operator algebra, dynamical systems, and free probability. Perhaps, our approach provides a new tool to study them.

Let $G_{N}$ be the one-vertex- $N$-loop-edge graph with

$$
V\left(G_{N}\right)=\{v\} \text { and } E\left(G_{N}\right)=\left\{e_{j}=v e_{j} v: j=1, \ldots, N\right\},
$$


where $N \in \mathbb{N}$. In [6], we showed that a graph von Neumann algebra $\mathbb{M}_{G_{N}}=$ $M \times_{\alpha} \mathbb{G}_{N}$ is $*$-isomorphic to the classical crossed product algebra $\mathcal{M}_{N}=$ $M \times_{\lambda} F_{N}$, where $M$ is a given von Neumann algebra and $\mathbb{G}_{N}$ is the graph groupoid of $G_{N}$, and where $F_{N}$ is the free group with $N$-generators. Here the group action $\lambda: F_{N} \rightarrow$ Aut $M$ satisfies that $\lambda_{g}(m)=\alpha_{w_{g}}(m)$ for all $m \in M, g=i_{1} \cdots i_{n} \in F_{N}$, and $w_{g}=w_{i_{1}} \cdots w_{i_{n}} \in \mathbb{G}_{N}$, where Aut $M$ is the automorphism group of $M$. Since the graph groupoid $\mathbb{G}_{N}$ of $G_{N}$ is a well-defined group and it is group-isomorphic to $F_{N}$, the von Neumann algebras $\mathbb{M}_{G_{N}}$ and $\mathcal{M}_{N}$ are indeed $*$-isomorphic.

Let $E: \mathbb{M}_{G} \rightarrow M$ be the canonical conditional expectation (since $\mathbb{D}_{G}=$ $\left.M \cdot L_{v}=M\right)$ and let $\operatorname{tr}: M \rightarrow \mathbb{C}$ be the faithful trace making the pair $(M, \operatorname{tr})$ be a faithful tracial $W^{*}$-probability space. Since $\mathbb{D}_{G}=M$, the trace $\operatorname{Tr}$ on $\mathbb{D}_{G}$ is identical to $\operatorname{tr}$ on $M$. So, we can determine the linear functional $\tau: \mathbb{M}_{G} \rightarrow \mathbb{C}$ by $\tau=\operatorname{tr} \circ E=\operatorname{tr} \circ E$. Let $F: \mathcal{M}_{N} \rightarrow M$ be the conditional expectation defined by

$$
F\left(\sum_{g \in F_{N}} m_{g} g\right)=m_{e} e \text { for all } \sum_{g \in F_{N}} m_{g} g \in \mathcal{M}_{N},
$$

where $e$ is the group-identity of $F_{N}$. If we define a continuous linear functional $\tau_{0}=\operatorname{tr} \circ F$ on $\mathcal{M}_{N}$, then the pairs $\left(\mathbb{M}_{G}, \tau\right)$ and $\left(\mathcal{M}_{N}, \tau_{0}\right)$ are equivalent (or identically distributed), in the sense of Voiculescu: (i) $\mathcal{M}_{N}$ and $\mathbb{M}_{G}$ are *isomorphic via a $*$-isomorphism $\Phi: \mathbb{M}_{G} \rightarrow \mathcal{M}_{N}$ and (ii) $\tau(x)=\tau_{0}(\Phi(x)$ ) for all $x \in \mathbb{M}_{G}$. Clearly, the $*$-isomorphism $\Phi$ is the generator-preserving map $m L_{w} \mapsto m g_{w}$, where $g_{w} \in F_{N}$ means the unique corresponding group element of $w \in \mathbb{G}_{N}$, for all $m \in M$. Remark that the pairs $\left(\mathbb{M}_{G}, E\right)$ and $\left(\mathcal{M}_{N}, F\right)$ are equivalent with amalgamation over $M$, too.

Suppose $M=\mathbb{C}$. Then the graph von Neumann algebra $M_{G_{N}}=\overline{\mathbb{C}\left[\mathbb{G}_{N}\right]}$ w of $G_{N}$ is determined, and it is $*$-isomorphic to the free group factor $L\left(F_{N}\right)$, because ${\overline{\mathbb{C}}\left[\mathbb{G}_{N}\right]}^{w}$ and $\overline{\mathbb{C}\left[F_{N}\right]}$ are $*$-isomorphic on $l^{2}\left(F_{N}\right)=H_{G_{N}}$ (See [6] and $[7])$. The diagonal tracial graph $W^{*}$-probability space $\left(M_{G_{N}}, \tau\right)$ is equivalent to $\left(L\left(F_{N}\right), \tau_{0}\right)$, where $\tau_{0}$ is the canonical trace on $L\left(F_{N}\right)$, defined by

$$
\tau_{0}\left(\sum_{g \in F_{N}} t_{g} g\right)=t_{e_{G}} \text { for all } \sum_{g \in F_{N}} t_{g} g \in L\left(F_{N}\right) .
$$

So, the study of the free probabilistic properties of $\left(M_{G_{N}}, \tau\right)$ is to study the free group factor $L\left(F_{N}\right)$. Furthermore, the $\mathbb{C}$-diagonal graph $W^{*}$-probability space $\left(M_{G_{N}}, E\right)$ and the diagonal tracial graph $W^{*}$-probability space $\left(M_{G_{N}}\right.$, $\tau)$ are identical, because the $\mathbb{C}$-diagonal subalgebra $D_{G_{N}}$ is nothing but $\mathbb{C}$, i.e., the pairs $\left(M_{G_{N}}, E\right)$ and $\left(M_{G_{N}}, \tau\right)$ are identically same.

\section{References}

[1] B. Bollobás, Modern Graph Theory, Graduate Texts in Mathematics, 184. SpringerVerlag, New York, 1998. 
[2] I. Cho, Graph-matrices over additive graph groupoids, Submitted to JAMC.

[3] _ Group-freeness and certain amalgamated freeness, J. Korean Math. Soc. 45 (2008), no. 3, 597-609.

$4]$, Direct producted $W^{*}$-probability spaces and corresponding amalgamated free stochastic integration, Bull. Korean Math. Soc. 44 (2007), no. 1, 131-150.

[5] $ـ$ Measures on graphs and groupoid measures, Complex Anal. Oper. Theory 2 (2008), no. 1, 1-28

[6] _ Graph von Neumann algebras, Acta Appl. Math. 95 (2007), no. 2, 95-134.

[7] Characterization of amalgamated free blocks of a graph von Neumann algebra, Complex Anal. Oper. Theory 1 (2007), no. 3, 367-398.

[8] - Vertex-compressed algebras of a graph von Neumann algebra, Acta Appl. Math. (2008), To Appear.

[9] - Operator Algebraic Quotient Structures of Graph von Neumann Algebras, CAOT, (2008), To Appear.

[10] I. Cho, and P. E. T. Jorgensen, $C^{*}$-algebras generated by partial isometries, JAMC, (2008), To Appear.

[11] —,$C^{*}$-subalgebras generated by partial isometries, JMP, (2008), To Appear.

[12] _ - Applications in automata and graphs: Labeling operators in Hilbert space I, (2007) Submitted to Acta Appl. Math: Special Issues.

[13] __ Applications in automata and graphs: Labeling operators in Hilbert space II, (2008) Submitted to JMP.

[14],$C^{*}$-subalgebras generated by a single operator in $B(H),(2008)$ Submitted to Acta Appl. Math: Special Issues.

[15] _ $C^{*}$-dynamical systems induced by partial isometries, (2008) Preprint.

[16] R. Diestel, Graph Theory: 3-rd edition, Graduate Texts in Mathematics, 173. SpringerVerlag, Berlin, 2005.

[17] R. Exel, Interaction, (2004) Preprint.

[18] _ A new look at the crossed-product of a $C^{*}$-algebra by an endomorphism, Ergodic Theory Dynam. Systems 23 (2003), no. 6, 1733-1750.

[19] R. Gliman, V. Shpilrain, and A. G. Myasnikov, Computational and Statistical Group Theory, Contemporary Mathematics, 298. American Mathematical Society, Providence, RI, 2002.

[20] V. F. R. Jones, Subfactors and Knots, CBMS Regional Conference Series in Mathematics, 80. Published for the Conference Board of the Mathematical Sciences, Washington, DC; by the American Mathematical Society, Providence, RI, 1991.

[21] M. T. Jury and D. W. Kribs, Ideal structure in free semigroupoid algebras from directed graphs, J. Operator Theory 53 (2005), no. 2, 273-302.

[22] A. G. Myasnikov and V. Shapilrain, Group Theory, Statistics and Cryptography, Contemporary Mathematics, 360. American Mathematical Society, Providence, RI, 2004.

[23] A. Nica, R-transform in Free Probability, IHP course note, available at www.math. uwaterloo.ca/ ${ }^{\sim}$ anica.

[24] A. Nica, D. Shlyakhtenko, and R. Speicher, R-cyclic families of matrices in free probability, J. Funct. Anal. 188 (2002), no. 1, 227-271.

[25] A. Nica and R. Speicher, R-diagonal Pair-A Common Approach to Haar Unitaries and Circular Elements, www.mast.queensu.ca/ ${ }^{\sim}$ speicher.

[26] F. Rădulescu, Random matrices, amalgamated free products and subfactors of the von Neumann algebra of a free group, of noninteger index, Invent. Math. 115 (1994), no. 2, 347-389.

[27] I. Raeburn, Graph Algebras, CBMS Regional Conference Series in Mathematics, 103. Published for the Conference Board of the Mathematical Sciences, Washington, DC; by the American Mathematical Society, Providence, RI, 2005. 
[28] B. Solel, You can see the arrows in a quiver operator algebra, J. Aust. Math. Soc. 77 (2004), no. 1, 111-122.

[29] R. Speicher, Combinatorial theory of the free product with amalgamation and operatorvalued free probability theory, Mem. Amer. Math. Soc. 132 (1998), no. 627, x+88 pp.

[30] _ Combinatorics of free probability theory IHP course note, available at www. mast.queensu.ca/ ${ }^{\sim}$ speicher.

[31] D. Voiculescu, Operations on certain non-commutative operator-valued random variables, Astérisque No. 232 (1995), 243-275.

[32] D. Voiculescu, K. Dykemma, and A. Nica, Free Random Variables, CRM Monograph Series, 1. American Mathematical Society, Providence, RI, 1992.

Department of Mathematics

SAint Ambrose University

$421 \mathrm{AH}, 518 \mathrm{~W}$. Locust St.

DAVENPORT, IOWA 52803, USA

E-mail address: chowoo@sau.edu 